University of Nebraska - Lincoln

DigitalCommons@University of Nebraska - Lincoln

\title{
Changes in Breeding Bird Populations in North Dakota: 1967 to 1992-93
}

Lawrence Igl

USGS Northern Prairie Wildlife Research Center, ligl@usgs.gov

Douglas Johnson

USGS Northern Prairie Wildlife Research Center, Douglas_H_Johnson@usgs.gov

Follow this and additional works at: https://digitalcommons.unl.edu/usgsnpwrc

Part of the Other International and Area Studies Commons

Igl, Lawrence and Johnson, Douglas, "Changes in Breeding Bird Populations in North Dakota: 1967 to 1992-93" (1997). USGS Northern Prairie Wildlife Research Center. 31.

https://digitalcommons.unl.edu/usgsnpwrc/31

This Article is brought to you for free and open access by the US Geological Survey at DigitalCommons@University of Nebraska - Lincoln. It has been accepted for inclusion in USGS Northern Prairie Wildlife Research Center by an authorized administrator of DigitalCommons@University of Nebraska - Lincoln. 


\title{
CHANGES IN BREEDING BIRD POPULATIONS IN NORTH DAKOTA: 1967 TO 1992-93
}

\author{
LAWRENCE D. IGL ${ }^{1}$ and DOUGLAS H. JoHNSON \\ National Biological Service, Northern Prairie Science Center, \\ Jamestown, North Dakota 58401, USA
}

\begin{abstract}
We compared breeding bird populations in North Dakota using surveys conducted in 1967 and 1992-93. In decreasing order, the five most frequently occurring species were Horned Lark (Eremophila alpestris), Brown-headed Cowbird (Molothrus ater), Western Meadowlark (Sturnella neglecta), Red-winged Blackbird (Agelaius phoeniceus), and Eastern Kingbird (Tyrannus tyrannus). The five most abundant species-Horned Lark, Chestnut-collared Longspur (Calcarius ornatus), Red-winged Blackbird, Western Meadowlark, and Brown-headed Cowbird-accounted for $31-41 \%$ of the estimated statewide breeding bird population in the three years. Although species composition remained relatively similar among years, betweenyear patterns in abundance and frequency varied considerably among species. Data from this survey and the North American Breeding Bird Survey indicated that species exhibiting significant declines were primarily grassland- and wetland-breeding birds, whereas species exhibiting significant increases primarily were those associated with human structures and woody vegetation. Population declines and increases for species with similar habitat associations paralleled breeding habitat changes, providing evidence that factors on the breeding grounds are having a detectable effect on breeding birds in the northern Great Plains. Received 30 January 1996, accepted 18 September 1996.
\end{abstract}

THE DECLINE OF BREEDING BIRD POPULATIONS in forests of eastern North America has received considerable recent attention (Askins et al. 1990, Hagan and Johnston 1992, Finch and Stangel 1993). The status of bird populations in grasslands and midcontinental areas, however, has received far less attention. Yet, analyses of North American Breeding Bird Survey (BBS) data indicate that more breeding species are declining than increasing in the prairie regions of North America (Droege and Sauer 1994). Also, grassland species show greater and more consistent patterns of decline at the continental level than do other ecological guilds, including long-distance migrants in eastern forests (Askins 1993, Droege and Sauer 1994). These declines have been most pronounced in areas of intensive agriculture, such as the Midwest (Herkert 1991, 1995; Warner 1994) and the northern Great Plains (Johnson and Schwartz 1993, Reynolds et al. 1994).

Although the BBS is the best source of quantitative data on trends of breeding bird populations in the midcontinent, it has several limitations, including sparse coverage in central North America and biases associated with road-

\footnotetext{
${ }^{1}$ E-mail: larry_igl@nbs.gov
}

side surveys. Several studies have attempted to verify population trends from the $B B S$ with data from independent, long-term surveys (e.g. Breeding Bird Atlas: Robbins et al. 1989; checklists: Temple and Cary 1990; Christmas Bird Count: Hagan 1993; migration counts: Dunn and Hussell 1995). Parallel trends derived from studying the same populations in different ways may provide corroborating evidence and strengthen the assessment of population trends of the BBS. Compared with eastern deciduous forests (e.g. Johnston and Hagan 1992), however, long-term data sets on breeding birds in the midcontinent are scarce.

Though a paucity of comparative information exists in the midcontinent, historic surveys provide an often overlooked source of baseline data on breeding bird populations (e.g. Graber and Graber 1963). These include the extensive survey of breeding birds in North Dakota conducted by Stewart and Kantrud (1972) in 1967 to obtain estimates of statewide breeding bird abundances and frequencies of occurrence. Data from the Stewart-Kantrud survey provided a unique opportunity to evaluate changes in breeding bird populations in the northern Great Plains. In 1992 and 1993, we repeated the Stewart-Kantrud survey using the same sample units and methods. Our objectives in this paper are 


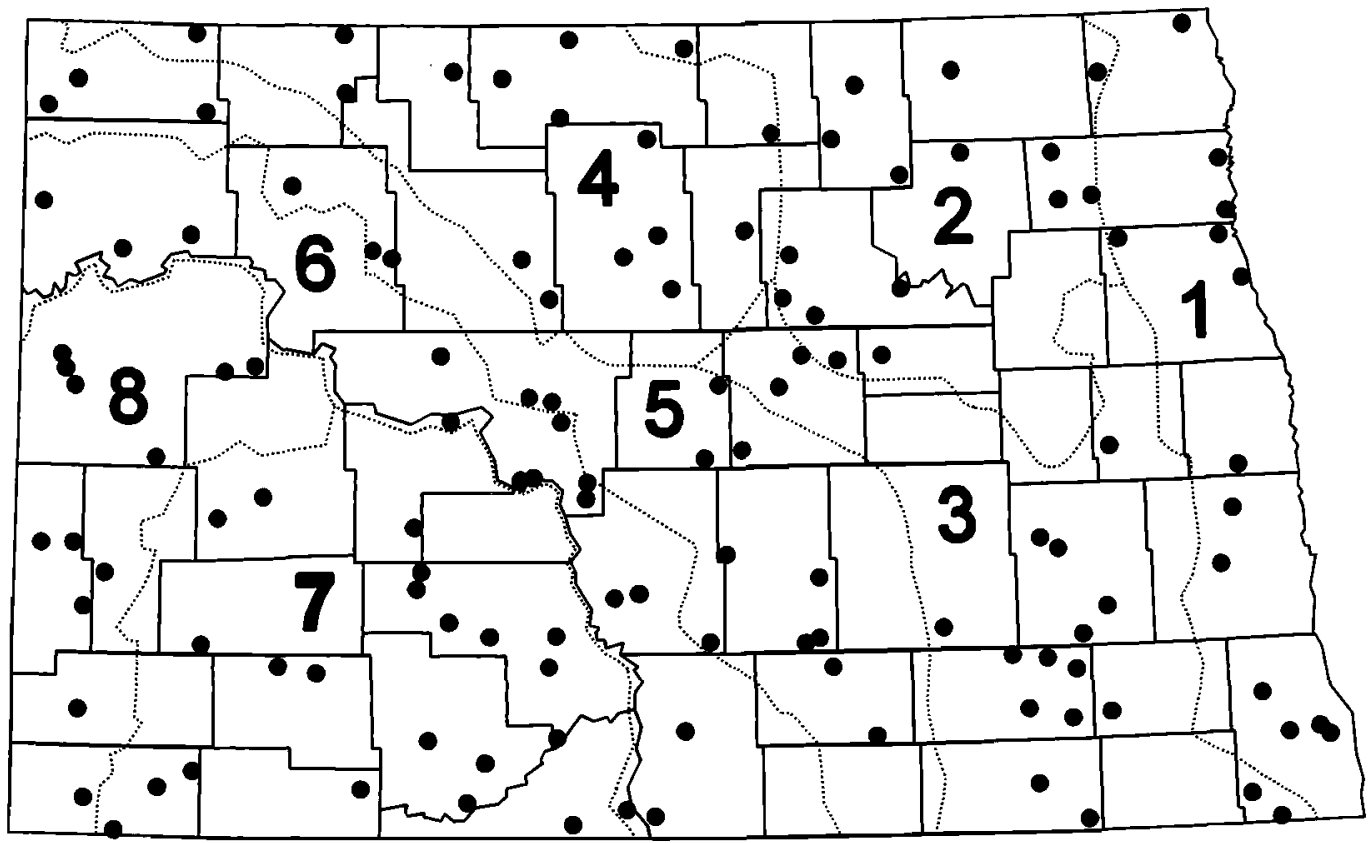

Fig. 1. Distribution of 128 quarter-sections in North Dakota where bird surveys were conducted during 1967 and 1992-93. Strata are indicated by dashed lines: (1) Agassiz Lake Plain, (2) Northeastern Drift Plain, (3) Southern Drift Plain, (4) Northwestern Drift Plain, (5) Missouri Coteau, (6) Coteau Slope, (7) Missouri Slope, and (8) Little Missouri Slope.

to: (1) examine changes in breeding bird populations in North Dakota; (2) compare patterns in breeding bird population changes with trends from the BBS; and (3) assess the likelihood that population changes have been influenced by changes in land use in North Dakota.

\section{Study AREA AND Methods}

Study area. - Situated in the geographical center of North America, North Dakota has a climate characterized by warm summers and long, cold winters. The distribution of precipitation is highly seasonal, with about $75 \%$ of the annual precipitation occurring during the growing season (April-September; Jensen 1972). Precipitation for January through June $(\bar{x}=$ $22.4 \mathrm{~cm}, 1961-1990)$ in North Dakota was nearly nor$\mathrm{mal}$ in $1967(21.8 \mathrm{~cm})$, below normal in $1992(18.0 \mathrm{~cm})$, and above normal in 1993 (25.7 cm; NOAA 1967, 1992, 1993). Many areas in North Dakota and the northern Great Plains experienced moderate to extreme drought conditions from 1988 to early 1993 (NOAA 1988-1993, Igl and Johnson 1995).

In 1967, Stewart and Kantrud (1972) divided the state into eight major strata based on biogeographical, physiographical, and ecological characteristics (Fig. 1). From these eight strata, Stewart and Kantrud (1972) selected 130 sample units by random selection without replacement. The number of sample units allocated to each stratum was proportional to the area of the stratum. Within each stratum, sample units were proportionately distributed according to the relative size of substrata that were differentiated on the basis of prevalent habitat types. The number of substrata ranged from two to five for each of the eight major strata and totaled 27 for the state. The stratification used by Stewart and Kantrud (1972) was effective in reducing the estimated variance in population estimates by as much as $15 \%$ compared with simple random sampling (Nelms et al, 1994).

To facilitate a direct comparison, we surveyed the same sample units used by Stewart and Kantrud (Fig. 1; H. A. Kantrud unpubl. data). We visited 128 of the 130 quarter-sections (each ca. 64.7 ha) originally surveyed by Stewart and Kantrud in 1967; landowners denied access to the two other quarter-sections. Comparisons among years are based on the 128 quartersections that were surveyed in all three years.

Methods.-We surveyed breeding birds using the same methods employed by Stewart and Kantrud (1972; H. A. Kantrud pers. comm.). Surveys were conducted by two observers on foot. Each observer surveyed a rectangular half $(805 \times 402 \mathrm{~m} ; 32.37 \mathrm{ha})$ of a quarter-section by following a standardized survey route. This route was $100 \mathrm{~m}$ inside of and parallel to 
the boundary of the rectangle. Deviations up to 100 $m$ from the route often were necessary to survey all habitats adequately. The rectangular halves usually were surveyed simultaneously, and an interval of 400 $m$ was maintained between observers. Both observers compared field notes at the end of each coverage of a sample unit to prevent duplications in the counts of wide-ranging birds such as vultures, hawks, and crows. We minimized observer bias in 1992 and 1993 by using the same two observers in both years. In 1993, some of the quarter-sections were surveyed by a single, experienced observer, who censused both rectangular halves on the same day.

Large wetlands required a different type of coverage. Birds on open water were counted with a spotting scope from the shoreline. In large zones of emergent vegetation, one observer attempted to flush large (e.g. ducks and herons) or secretive (e.g. rails and bitterns) species by wading in a zigzag course throughout the wetland while making noise. From a nearby vantage point, the second observer recorded all birds flushed, including conspicuous, colonial marsh birds such as Red-winged Blackbirds (Agelaius phoeniceus).

The phenological advance in seasons during the spring and early summer is about two weeks earlier in southwestern North Dakota than in the northeastern portion of the state. To compensate for these differences, the sequence in which sample units were covered progressed from southwestern to northeastern North Dakota. We matched the date that a quartersection was surveyed in 1992 and 1993 as closely as feasible to the date that it was surveyed in 1967 ( $\mathrm{H}$. A. Kantrud unpubl. data). The surveys of breeding birds extended from 24 April to 19 July in 1967, from 27 April to 18 July in 1992, and from 24 April to 21 July in 1993. The overall absolute difference between the 1967 surveys and the 1992 and 1993 surveys averaged 3.3 days and 1.7 days, respectively.

In each year, sample units were surveyed once or twice; the number of breeding pairs for each species, however, was based on single counts during each species' peak breeding period. All sample units were surveyed for early-nesting species between 24 April and 7 June, for mid-nesting species between 14 May and $10 \mathrm{July}$, and for late-nesting species between 22 May and 21 July (Table 1). When a survey was conducted during an overlapping portion of the peak breeding periods, counts of early-, mid-, and latenesting species coincided. Thus, quarter-sections that were visited between 22 May to 7 June were surveyed only once, and those that were surveyed before 22 May were surveyed again after 7 June to include species from all three breeding periods. Peak breeding periods for some species differ from those in Stewart and Kantrud (1972) due to ty pographical errors in the original publication; these errors did not affect statewide population estimates or variances in Stewart and Kantrud (1972). Stewart and Kantrud (1972, Kantrud
1982) felt justified in estimating bird populations in open habitats using single counts because many species have behavioral adaptations (e.g. elevated perches, flight songs, synchronous displays) that tend to increase their detectability compared with birds inhabiting extensive wooded areas (see Speirs and Orenstein 1967, Cody 1985).

Species were identified by sight or sound. Counts during precipitation and strong winds $(>24 \mathrm{~km} / \mathrm{h})$ were avoided. Surveys of open-country birds were conducted between $0.5 \mathrm{~h}$ after sunrise and $0.5 \mathrm{~h}$ before sunset. Although some surveys occurred outside the time of peak vocal activity (i.e. early morning or late evening), Stewart and Kantrud (1972) concluded that singing and other activities of open-country birds were not appreciably affected by time of day. Quarter-sections containing extensive woodland habitats usually were covered on relatively calm $(<8 \mathrm{~km} / \mathrm{h})$, sunny days between $0.5 \mathrm{~h}$ after sunrise and $1000 \mathrm{CST}$. These limitations were necessary because song frequencies and other activities of most woodland birds are reduced on cloudy days, in moderate or high winds, and at midday.

Counts of breeding birds were based primarily on the number of indicated breeding pairs on territories or home ranges during peak breeding periods. For most species, nearly all indicated pairs were observed as segregated pairs or as territorial males. For Wilson's Phalarope (Phalaropus tricolor), segregated pairs and lone females were recorded as indicated pairs. Although currently not in vogue, for consistency we based the number of indicated pairs of Brown-headed Cowbird (Molothrus ater) on the total number of males seen per sample unit. In the case of colonial birds that are not sexually dimorphic (e.g. Black Tern [Chlidonias niger] and Cliff Swallow [Hirundo pyrrhonota]), the number of indicated pairs was based either on a count of occupied nests or was derived by halving the total number of individuals counted.

The procedures used to determine the number of pairs of breeding waterfowl followed Hammond (1969) with one exception. Occasionally, the number of lone females on a given quarter-section exceeded the number of males unaccompanied by females. In this case, each excess lone female was considered to represent an indicated pair.

We excluded from our results birds that we considered to be nonbreeders. These included: (1) migrant flocks and individuals of species that are not known to breed in North Dakota (Faanes and Stewart 1982); (2) nonbreeding, vagrant waterbirds in the summer and oversummering shorebirds (i.e. transient shorebirds remaining in North Dakota during the boreal summer); (3) wide-ranging colonial waterbirds passing high overhead (e.g. pelicans and gulls); and (4) other birds passing overhead in high, direct flight. By counting birds only during their peak breeding periods, we maximized the potential for recording breeding pairs and territorial males and, at the same 
time, minimized the likelihood for confounding territorial birds with migrants.

Vernacular and scientific names follow the American Ornithologists' Union (1983) and subsequent supplements, with one exception. We recorded Redshafted (Colaptes auratus cafer) and Yellow-shafted ( $C$. auratus auratus) subspecies of the Northern Flicker separately to reflect their species status in 1967. One obvious intergrade was recorded as a Red-shafted Flicker in 1967.

Species classification.-Based on published accounts, we classified each species into one of three groups according to its migratory behavior (Table 1): permanent resident (present in North Dakota year-round), short-distance migrant (winters primarily north of the U.S./Mexico border), and long-distance migrant (winters primarily south of the U.S./Mexico border; Faanes and Stewart 1982, AOU 1983, Harrison 1983, Rappole et al. 1983, Hayman et al. 1986, Madge and Burn 1988, Thompson et al. 1993). The migratory status of some year-round residents (e.g. Blue Jay [Cyanocitta cristata], American Crow [Corvus brachyrhynchos], European Starling [Sturnus vulgaris]) was difficult to determine because some wintering individuals may have originated from breeding populations north of North Dakota. We considered these species to be short-distance migrants. In addition, we categorized each species into a general breeding habitat based on the literature (Ehrlich et al. 1988, Peterjohn and Sauer 1993) and personal observations. Habitats were described as wetland (including wet meadow), grassland, open habitat with scattered trees, woodland, open or semiopen deciduous woodland and edge, shrubland, residential areas and human-made structures (hereafter, human-made structures), and "other" (mostly unvegetated habitats including clay buttes, cliffs, banks, etc.). Waterfowl were included in the wetland class, i.e. their primary foraging and broodrearing habitat. Species using a broad range of habitats (e.g. Song Sparrow [Melospiza melodia], Common Yellowthroat [Geothlypis trichas]) were classified according to their principal habitat type.

Calculation of population estimates. -We estimated population means and totals, and their standard deviations, using standard methods for stratified random samples with proportional allocation (Cochran 1977). We calculated Bayesian confidence intervals (95\% confidence limits; Box and Tiao 1973) in lieu of the usual confidence intervals using the methods described in Johnson (1977). Bayesian intervals exploit the prior knowledge that the means of bird densities and of total numbers of birds are non-negative. Population estimates are given only for species with statewide frequencies of occurrence of $10 \%$ or higher (i.e. common species). Results for 1967 in this paper may vary slightly from those given in Stewart and Kantrud (1972) due to differences in sample size (i.e. 130 vs. 128 quarter-sections).

Statewide population estimates were compared be- tween 1967 and 1992-93 with z-tests. A significant change was claimed only if the difference between 1967 and 1992 values and the difference between 1967 and 1993 values were both significant at $P \leq 0.10$ and only if both differences were in the same direction.

Biases associated with the bird survey were not quantified (see Stewart and Kantrud 1972). In 1967, Stewart and Kantrud made efforts to minimize apparent biases in methodology through adjustments in census techniques. In the recent surveys, we endeavored to conduct our surveys as similarly as possible to the methods used in 1967 . We recognize that the size of the breeding population for certain species may be over- or underestimated. For example, we assumed that all males were mated, although some territorial males may have been unmated (e.g. Dickcissel [Spiza americana], Fretwell and Calver 1970; Ovenbird [Seiurus aurocapillus], Gibbs and Faaborg 1990). Also, population estimates of wide-ranging species or species with large territories or home ranges may have been overestimated. For polygynous (e.g. Yellow-headed Blackbird [Xanthocephalus xanthocephalus]) and polyandrous (Wilson's Phalarope) species, the number of indicated pairs represents, in terms of breeding mates, a minimum population. Undoubtedly, biases related to differences in observers, years, weather, sampling time, etc. were present, but biases associated with methodology should be relatively consistent among years.

Breeding Bird Survey trends. - We obtained trends in abundance from the BBS for North Dakota during the period 1967-1993 (J. R. Sauer and B. G. Peterjohn pers. comm.). Trends are based on statistical methods described by Geissler and Sauer (1990) and are presented as the average percent annual change between 1967 and 1993. In North Dakota, the BBS began in 1967, the same year that Stewart and Kantrud (1972) conducted their survey.

Land use changes. - Comparable data on land use and cover were available each year for every quarter-section. To evaluate overall changes in major habitats, we digitized land use and cover by drawing vectors over scaled rasters of scanned aerial photographs using Map and Image Processing System (MIPS) software (Microlmages, Inc. 1992). Eight major land use or cover classes were delineated: (1) Cropland (all land used for the production of annual field crops and land under summer fallow); (2) Hayland (areas that have been plowed and seeded to mixtures of grasses and legumes for forage or seed production); (3) Grassland (natural grassland regardless of condition and disturbance regime [e.g. grazed, mowed, idle] and areas planted to introduced species); (4) Planted Cover (mixtures of grasses and legumes planted for wildlife cover or soil conservation [e.g. Conservation Reserve Program]); (5) Woodland and Shrubland (native and artificially stocked tree and shrub stands and plantings); (6) Wetland (areas classified as wetlands by Stewart and Kantrud [1971] as well as permanent 
TABLE 1. Breeding bird populations in 128 randomly selected quarter-sections in North Dakota.

\begin{tabular}{|c|c|c|c|c|c|c|c|}
\hline \multirow[b]{2}{*}{ Species $^{a}$} & \multicolumn{3}{|c|}{ No. indicated pairs } & \multirow{2}{*}{$\begin{array}{l}\text { Migratory } \\
\text { status }^{\mathbf{b}}\end{array}$} & \multirow{2}{*}{$\begin{array}{l}\text { Breeding } \\
\text { habitat }^{c}\end{array}$} & \multirow{2}{*}{$\begin{array}{l}\text { Popula- } \\
\text { tion } \\
\text { change }^{d}\end{array}$} & \multirow{2}{*}{$\begin{array}{l}\text { BBS } \\
\text { trend }^{\mathrm{e}}\end{array}$} \\
\hline & 1967 & 1992 & 1993 & & & & \\
\hline Pied-billed Grebe (Podilymbus podiceps) ${ }^{* * *}$ & 11 & 4 & 7 & SDM & WETL & NC & $-7.55 \downarrow \downarrow$ \\
\hline Horned Grebe (Podiceps auritus) ${ }^{* * *}$ & 2 & 1 & 0 & SDM & WETL & NC & -0.22 \\
\hline Red-necked Grebe (Podiceps grisegena) ${ }^{* * *}$ & 0 & 1 & 1 & SDM & WETL & NC & NA \\
\hline Eared Grebe (Podiceps nigricollis) ${ }^{* * *}$ & 40 & 48 & 22 & SDM & WETL & NC & -1.28 \\
\hline Western Grebe (Aechmophorus occidentalis) ${ }^{* *}$ & 0 & 2 & 1 & SDM & WETL & NC & $-28.08 \downarrow \downarrow$ \\
\hline American White Pelican (Pelecanus erythrorhynchos)** & 0 & 0 & 2 & SDM & WETL & NC & $-6.45 \downarrow$ \\
\hline Double-crested Cormorant (Phalacrocorax auritus) ${ }^{* *}$ & 0 & 1 & 10 & SDM & WETL & NC & -3.51 \\
\hline American Bittern (Botaurus lentiginosus) ${ }^{* * *}$ & 9 & 2 & 8 & LDM & WETL & NC & $-3.54 \downarrow \downarrow \downarrow$ \\
\hline Great Blue Heron (Ardea herodias) ${ }^{* *}$ & 2 & 1 & 3 & SDM & WETL & $\mathrm{NC}$ & +1.00 \\
\hline Black-crowned Night-Heron (Nycticorax nycticorax) ${ }^{* * *}$ & $\overline{17}$ & 5 & 5 & SDM & WETL & NC & $-4.76 \downarrow \downarrow$ \\
\hline Canada Goose (Branta canadensis)* & 0 & 11 & 28 & SDM & WETL & NC & $+9.45 \uparrow \uparrow \uparrow$ \\
\hline Wood Duck (Aix sponsa) ${ }^{*}$ & 1 & 2 & 12 & SDM & WETL & NC & +0.80 \\
\hline Green-winged Teal (Anas crecca) $)^{* *}$ & 45 & 11 & 44 & SDM & WETL & NC & -0.64 \\
\hline Mallard (Anas platyrhynchos) ${ }^{*}$ & 212 & 113 & 200 & SDM & WETL & NC & +1.01 \\
\hline Northern Pintail (Anas acuta) & 171 & 23 & 58 & SDM & WETL & $\downarrow$ & -3.68 \\
\hline Cinnamon Teal (Anas cyanoptera)** & 0 & 1 & 0 & LDM & WETL & NC & NA \\
\hline Blue-winged Teal (Anas discors) ${ }^{* *}$ & 286 & 99 & 145 & LDM & WETL & $\downarrow$ & $-3.20 \downarrow \downarrow \downarrow$ \\
\hline Northern Shoveler (Anas clypeata) ${ }^{* *}$ & 87 & 25 & 52 & SDM & WETL & $\downarrow$ & +0.05 \\
\hline Gadwall (Anas strepera) ** & 96 & 111 & 118 & SDM & WETL & NC & +0.36 \\
\hline American Wigeon (Anas americana) ${ }^{* *}$ & 25 & 22 & 32 & SDM & WETL & NC & $-3.75 \downarrow \downarrow$ \\
\hline Canvasback (Aythya valisineria) ${ }^{* *}$ & 10 & 4 & 5 & SDM & WETL & NC & -1.58 \\
\hline Redhead (Aythya americana) ${ }^{* * *}$ & 27 & 19 & 42 & SDM & WETL & NC & -2.96 \\
\hline Ring-necked Duck (Aythya collaris) ${ }^{* *}$ & 2 & 0 & 1 & SDM & WETL & $\mathrm{NC}$ & $-10.88 \downarrow \downarrow \downarrow$ \\
\hline Lesser Scaup (Aythya affinis) ${ }^{* * *}$ & 3 & 4 & 4 & SDM & WETL & NC & -0.63 \\
\hline Bufflehead (Bucephala albeola $)^{* * *}$ & 0 & 0 & 1 & SDM & WETL & NC & $-4.65 \downarrow \downarrow$ \\
\hline Hooded Merganser (Lophodytes cucullatus)* & 0 & 0 & 2 & SDM & WETL & NC & $+0.91 \uparrow \uparrow \uparrow$ \\
\hline Ruddy Duck (Oxyura jamaicensis)*** & 36 & 56 & 23 & SDM & WETL & NC & -3.20 \\
\hline Turkey Vulture (Cathartes aura) ${ }^{* *}$ & 2 & 0 & 1 & SDM & OPWO & NC & +4.12 \\
\hline Northern Harrier (Circus cyaneus) $)^{* *}$ & 15 & 21 & 34 & SDM & GRAS & NC & +1.76 \\
\hline Sharp-shinned Hawk (Accipiter striatus) ${ }^{* *}$ & 0 & 1 & 0 & SDM & WOOD & NC & $-1.74 \downarrow$ \\
\hline Cooper's Hawk (Accipiter cooperii) ${ }^{* *}$ & 3 & 2 & 8 & SDM & WOOD & NC & $+3.76 \uparrow \uparrow \uparrow$ \\
\hline Broad-winged Hawk (Buteo platypterus) ${ }^{* *}$ & 0 & 1 & 1 & LDM & WOOD & NC & NA \\
\hline Swainson's Hawk (Buteo swainsoni) ${ }^{* *}$ & 7 & 14 & 25 & LDM & OPTR & $\uparrow$ & $+3.04 \uparrow \uparrow \uparrow$ \\
\hline Red-tailed Hawk (Buteo jamaicensis) ${ }^{*}$ & 7 & 11 & 16 & SDM & OPTR & $\mathrm{NC}$ & +1.61 \\
\hline Ferruginous Hawk (Buteo regalis) & 4 & 4 & 8 & SDM & GRAS & $\mathrm{NC}$ & +2.51 \\
\hline Golden Eagle (Aquila chrysaetos) ${ }^{*}$ & 0 & 2 & 3 & SDM & OTHR & $\uparrow$ & +0.80 \\
\hline American Kestrel (Falco sparverius) ${ }^{* *}$ & 5 & 10 & 11 & SDM & OPTR & NC & +3.37 \\
\hline Prairie Falcon (Falco mexicanus) $)^{* *}$ & 0 & 3 & 3 & SDM & OTHR & $\uparrow$ & +0.34 \\
\hline Gray Partridge (Perdix perdix) ${ }^{*}$ & 17 & 40 & 36 & RES & GRAS & $\uparrow$ & $+3.97 \uparrow \uparrow \uparrow$ \\
\hline
\end{tabular}




\begin{tabular}{|c|c|c|c|c|c|c|c|}
\hline \multirow[b]{2}{*}{ Species $^{\mathrm{a}}$} & \multicolumn{3}{|c|}{ No. indicated pairs } & \multirow{2}{*}{$\begin{array}{l}\text { Migratory } \\
\text { status }^{\mathrm{b}}\end{array}$} & \multirow{2}{*}{$\begin{array}{l}\text { Breeding } \\
\text { habitat }^{c}\end{array}$} & \multirow{2}{*}{$\begin{array}{l}\text { Popula- } \\
\text { tion } \\
\text { change }^{d}\end{array}$} & \multirow{2}{*}{$\begin{array}{c}\text { BBS } \\
\text { trend }^{\mathrm{e}}\end{array}$} \\
\hline & 1967 & 1992 & 1993 & & & & \\
\hline Ring-necked Pheasant (Phasianus colchicus) ${ }^{*}$ & 3 & 24 & 51 & RES & GRAS & $\uparrow$ & $+4.24 \uparrow \uparrow \uparrow$ \\
\hline Sharp-tailed Grouse (Tympanuchus phasianellus)* & 18 & 46 & 32 & RES & GRAS & $\uparrow$ & $+5.30 \uparrow \uparrow$ \\
\hline Wild Turkey (Meleagris gallopavo) & 0 & 0 & 3 & RES & OPWO & NC & $+6.66 \uparrow \uparrow \uparrow$ \\
\hline Yellow Rail (Coturnicops noveboracensis) ${ }^{* * *}$ & 0 & 1 & 0 & SDM & WETL & NC & $+0.93 \uparrow 1$ \\
\hline Virginia Rail (Rallus limicola) ${ }^{* * *}$ & 3 & 5 & 2 & SDM & WETL & NC & -0.37 \\
\hline Sora (Porzana carolina) ${ }^{* *}$ & 32 & 41 & $7 \overline{8}$ & SDM & WETL & $\mathrm{NC}$ & $-3.11 \downarrow \downarrow \downarrow$ \\
\hline American Coot (Fulica americana) ${ }^{* *}$ & 348 & 76 & 124 & SDM & WETL & $\downarrow$ & $-7.53 \downarrow \downarrow \downarrow$ \\
\hline Piping Plover (Charadrius melodus)** & 5 & 2 & 1 & SDM & WETL & NC & NA \\
\hline Killdeer (Charadrius vociferus) ${ }^{*}$ & 105 & 112 & 142 & SDM & WETL & $\mathrm{NC}$ & $-1.65 \downarrow$ \\
\hline American Avocet (Recurvirostra americana) ${ }^{* *}$ & 14 & 6 & 13 & SDM & WETL & $\mathrm{NC}$ & +0.19 \\
\hline Willet (Catoptrophorus semipalmatus) ${ }^{* *}$ & 18 & 16 & 27 & LDM & WETL & NC & -0.77 \\
\hline Spotted Sandpiper (Actitis macularia) ${ }^{* *}$ & 12 & 12 & 9 & LDM & WETL & NC & -0.33 \\
\hline Upland Sandpiper (Bartramia longicauda) ${ }^{* *}$ & 63 & 106 & 89 & LDM & GRAS & $\uparrow$ & $+4.85 \uparrow \uparrow \uparrow$ \\
\hline Marbled Godwit (Limosa fedoa)* & 17 & 8 & 14 & SDM & GRAS & $\mathrm{NC}$ & +1.66 \\
\hline Common Snipe (Gallinago gallinago)** & 0 & 2 & 7 & SDM & WETL & $\uparrow$ & +5.93 \\
\hline Wilson's Phalarope (Phalaropus tricolor) ${ }^{* *}$ & 73 & 30 & 36 & LDM & WETL & $\downarrow$ & $-6.43 \downarrow \downarrow \downarrow$ \\
\hline Franklin's Gull (Larus pipixcan)** & 22 & 79 & 56 & LDM & WETL & $\mathrm{NC}$ & $-9.11 \downarrow$ \\
\hline Ring-billed Gull (Larus delawarensis) ${ }^{* *}$ & 1 & 49 & 11 & SDM & WETL & $\uparrow$ & -5.25 \\
\hline California Gull (Larus californicus) ${ }^{* *}$ & 0 & 0 & 2 & SDM & WETL & NC & $-5.80 \downarrow \downarrow \downarrow$ \\
\hline Forster's Tern (Sterna forsteri) ${ }^{* *}$ & 3 & 6 & 4 & SDM & WETL & NC & -0.20 \\
\hline Common Tern (Sterna hirundo) ${ }^{* *}$ & 6 & 6 & 3 & LDM & WETL & NC & -0.00 \\
\hline Black Tern (Chlidonias niger) ${ }^{* * *}$ & 118 & 39 & 39 & LDM & WETL & $\downarrow$ & $-5.69 \downarrow$ \\
\hline Rock Dove (Columba livia) ${ }^{*}$ & 0 & 23 & 12 & RES & RESI & $\dot{\uparrow}$ & +1.60 \\
\hline Mourning Dove (Zenaida macroura) ${ }^{* *}$ & 292 & 339 & 337 & SDM & OPWO & NC & $+3.70 \uparrow \uparrow \uparrow$ \\
\hline Black-billed Cuckoo (Coccyzus erythropthalmus) ${ }^{* *}$ & 7 & 30 & 10 & LDM & WOOD & NC & +2.08 \\
\hline Great Horned Owl (Bubo virginianus)* & 2 & 4 & 9 & RES & OPWO & NC & $+2.88 \uparrow \uparrow \uparrow$ \\
\hline Burrowing Owl (Speotyto cunicularia) $)^{* *}$ & 3 & 3 & 2 & LDM & GRAS & NC & -0.86 \\
\hline Long-eared Owl (Asio otus $)^{* *}$ & 0 & 1 & 1 & SDM & OPWO & NC & NA \\
\hline Short-eared Owl (Asio flammeus)* & 0 & 1 & 1 & SDM & GRAS & NC & +1.65 \\
\hline Common Nighthawk (Chordeiles minor) ${ }^{* * *}$ & 10 & 12 & 16 & LDM & OPWO & $\mathrm{NC}$ & +0.58 \\
\hline Chimney Swift (Chaetura pelagica) $* * *$ & 1 & 0 & 3 & LDM & RESI & $\mathrm{NC}$ & $+5.21 \uparrow \uparrow$ \\
\hline Ruby-throated Hummingbird (Archilochus colubris) ${ }^{* * *}$ & 0 & 4 & 0 & LDM & OPWO & NC & NA \\
\hline Belted Kingfisher (Ceryle alcyon $)^{* *}$ & 0 & 1 & 1 & SDM & WETL & $\mathrm{NC}$ & -0.46 \\
\hline Red-headed Woodpecker (Melanerpes erythrocephalus) ${ }^{* *}$ & 2 & 9 & 7 & SDM & OPWO & $\uparrow$ & -0.70 \\
\hline Yellow-bellied Sapsucker (Sphyrapicus varius) ${ }^{* *}$ & 0 & 0 & 2 & SDM & WOOD & NC & +0.76 \\
\hline Downy Woodpecker (Picoides pubescens)** & 0 & 9 & 21 & RES & OPWO & $\uparrow$ & $+1.88 \uparrow \uparrow \uparrow$ \\
\hline Hairy Woodpecker (Picoides villosus) ${ }^{* *}$ & 2 & 12 & 11 & RES & OPWO & $\uparrow$ & +0.93 \\
\hline Yellow-shafted Flicker (Colaptes auratus auratus) ${ }^{* *}$ & 40 & 25 & 35 & SDM & OPWO & NC & $-3.85 \downarrow \downarrow \downarrow$ \\
\hline Red-shafted Flicker (Colaptes auratus cafer) * " & 12 & 5 & 4 & SDM & OPWO & NC & -0.82 \\
\hline
\end{tabular}




\begin{tabular}{|c|c|c|c|c|c|c|c|}
\hline \multirow[b]{2}{*}{ Species $^{\mathrm{a}}$} & \multicolumn{3}{|c|}{ No. indicated pairs } & \multirow{2}{*}{$\begin{array}{c}\text { Migratory } \\
\text { status }^{\mathrm{b}}\end{array}$} & \multirow{2}{*}{$\begin{array}{l}\text { Breeding } \\
\text { habitat }^{c}\end{array}$} & \multirow{2}{*}{$\begin{array}{l}\text { Popula- } \\
\text { tion } \\
\text { change }^{\text {d }}\end{array}$} & \multirow{2}{*}{$\begin{array}{c}\text { BBS } \\
\text { trend }\end{array}$} \\
\hline & 1967 & 1992 & 1993 & & & & \\
\hline Pileated Woodpecker (Dryocopus pileatus) ${ }^{* *}$ & 0 & 0 & 1 & RES & WOOD & NC & +1.12 \\
\hline Eastern Wood-Pewee (Contopus virens) $)^{* * *}$ & 2 & 10 & 7 & LDM & WOOD & NC & -1.46 \\
\hline Alder Flycatcher (Empidonax alnorum) ${ }^{* * *}$ & 0 & 0 & 1 & LDM & SHRU & NC & +3.34 \\
\hline Willow Flycatcher (Empidonax traillii) ${ }^{* * *}$ & 30 & 17 & 37 & LDM & SHRU & NC & +1.63 \\
\hline Least Flycatcher (Empidonax minimus) ${ }^{* * *}$ & 23 & 52 & 81 & LDM & OPWO & $\uparrow$ & +1.99 \\
\hline Eastern Phoebe (Sayornis phoebe) ${ }^{* * *}$ & 2 & 2 & 3 & SDM & OPWO & NC & +0.78 \\
\hline Say's Phoebe (Sayornis saya) ${ }^{* * *}$ & 6 & 4 & 17 & SDM & RESI & $\mathrm{NC}$ & $+2.51 \uparrow$ \\
\hline Great Crested Flycatcher (Myiarchus crinitus)*** & 3 & 8 & 13 & LDM & WOOD & NC & +1.18 \\
\hline Western Kingbird (Tyrannus verticalis) ${ }^{* *}$ & 103 & 194 & 177 & LDM & OPTR & $\uparrow$ & $+5.99 \uparrow \uparrow \uparrow$ \\
\hline Eastern Kingbird (Tyrannus tyrannus) ${ }^{* * *}$ & 167 & 322 & 245 & LDM & OPTR & $\uparrow$ & $+6.47 \uparrow \uparrow \uparrow$ \\
\hline Horned Lark (Eremophila alpestris) ${ }^{*}$ & 1253 & 1093 & 1661 & SDM & GRAS & NC & +0.30 \\
\hline Purple Martin (Progne subis) ${ }^{* * *}$ & 2 & 1 & 0 & LDM & RESI & NC & -0.01 \\
\hline Tree Swallow (Tachycineta bicolor) ${ }^{* *}$ & 3 & 5 & 12 & SDM & WETL & NC & +2.64 \\
\hline No. Rough-winged Swallow (Stelgidopteryx serripennis) ${ }^{* * *}$ & 7 & 1 & 3 & LDM & OTHR & NC & +0.81 \\
\hline Bank Swallow (Riparia riparia) ${ }^{* * *}$ & 77 & 112 & 61 & LDM & OTHR & NC & -0.30 \\
\hline Cliff Swallow (Hirundo pyrrhonota) ${ }^{* * *}$ & 152 & 343 & 226 & LDM & RESI & NC & $+7.23 \uparrow$ \\
\hline Barn Swallow (Hirundo rustica) ${ }^{* * *}$ & 96 & 187 & 194 & LDM & RESI & $\uparrow$ & $+2.31 \uparrow \uparrow \uparrow$ \\
\hline Blue Jay (Cyanocitta cristata) ${ }^{* * *}$ & 14 & 27 & 31 & SDM & OPWO & $\uparrow$ & +1.66 \\
\hline Black-billed Magpie (Pica pica) ${ }^{*}$ & 20 & 15 & 15 & RES & OPTR & NC & -8.36 \\
\hline American Crow (Corvus brachyrhynchos) ${ }^{*}$ & 28 & 13 & 27 & SDM & OPWO & $\mathrm{NC}$ & +0.26 \\
\hline Black-capped Chickadee (Parus atricapillus) ${ }^{* * *}$ & 3 & 28 & 31 & RES & OPWO & $\uparrow$ & +1.27 \\
\hline White-breasted Nuthatch (Sitta carolinensis) ${ }^{* * *}$ & 3 & 7 & 9 & RES & OPWO & $\mathrm{NC}$ & -0.61 \\
\hline Rock Wren (Salpinctes obsoletus) ${ }^{* * *}$ & 10 & 23 & 27 & SDM & OTHR & $\uparrow$ & +8.64 \\
\hline House Wren (Troglodytes aedon) ${ }^{* * *}$ & 52 & 219 & 209 & LDM & OPWO & $\uparrow$ & $+3.55 \uparrow \uparrow \uparrow$ \\
\hline Sedge Wren (Cistothorus platensis) ${ }^{* * *}$ & 10 & 20 & 37 & SDM & GRAS & NC & +0.95 \\
\hline Marsh Wren (Cistothorus palustris) $)^{* * *}$ & 51 & 113 & 153 & SDM & WETL & NC & +5.02 \\
\hline Eastern Bluebird (Sialia sialis) ${ }^{* *}$ & 0 & 4 & 5 & SDM & OPWO & $\uparrow$ & +0.48 \\
\hline Mountain Bluebird (Sialia currucoides) ${ }^{* *}$ & 8 & 15 & 20 & SDM & OPWO & $\mathrm{NC}$ & +3.88 \\
\hline Veery (Catharus fuscescens) ${ }^{* *}$ & 11 & 5 & 3 & LDM & WOOD & NC & -2.99 \\
\hline American Robin (Turdus migratorius) ${ }^{* *}$ & 67 & 117 & 123 & SDM & OPWO & $\uparrow$ & $+3.51 \uparrow \uparrow \uparrow$ \\
\hline Gray Catbird (Dumetella carolinensis) ${ }^{* *}$ & 48 & 44 & 38 & LDM & SHRU & NC & -1.14 \\
\hline Brown Thrasher (Toxostoma rufum) ${ }^{* * *}$ & 42 & 85 & 104 & SDM & SHRU & $\uparrow$ & $+2.19 \uparrow \uparrow \uparrow$ \\
\hline Sprague's Pipit (Anthus spragueii)*" & 7 & 13 & 19 & SDM & GRAS & NC & $-2.43 \downarrow \downarrow$ \\
\hline Cedar Waxwing (Bombycilla cedrorum) $)^{* *}$ & 23 & 129 & 43 & SDM & OPWO & NC & +3.20 \\
\hline Loggerhead Shrike (Lanius ludovicianus) ${ }^{* *}$ & 8 & 16 & 15 & SDM & SHRU & $\uparrow$ & -0.41 \\
\hline European Starling (Sturnus vulgaris) ${ }^{*}$ & 12 & 47 & 40 & SDM & RESI & NC & +0.18 \\
\hline Yellow-throated Vireo (Vireo flavifrons) ${ }^{* * *}$ & 0 & 0 & 2 & LDM & WOOD & NC & +1.65 \\
\hline Warbling Vireo (Vireo gilvus) ${ }^{* * *}$ & 19 & 23 & 41 & LDM & OPWO & NC & $+5.06 \uparrow \uparrow \uparrow$ \\
\hline Red-eyed Vireo (Vireo olivaceus) ${ }^{* * *}$ & 13 & 20 & 42 & LDM & WOOD & NC & +0.19 \\
\hline
\end{tabular}


TABLE 1. Continued.

\begin{tabular}{|c|c|c|c|c|c|c|c|}
\hline \multirow[b]{2}{*}{ Species $^{a}$} & \multicolumn{3}{|c|}{ No. indicated pairs } & \multirow{2}{*}{$\begin{array}{c}\text { Migratory } \\
\text { status }^{\mathrm{b}}\end{array}$} & \multirow{2}{*}{$\begin{array}{l}\text { Breeding } \\
\text { habitat }^{*}\end{array}$} & \multirow{2}{*}{$\begin{array}{l}\text { Popula- } \\
\text { tion } \\
\text { change }^{d}\end{array}$} & \multirow{2}{*}{$\begin{array}{c}\text { BBS } \\
\text { trend }^{e}\end{array}$} \\
\hline & 1967 & 1992 & 1993 & & & & \\
\hline Black-and-white Warbler (Mniotilta varia) ${ }^{* * *}$ & 3 & 7 & 21 & LDM & OPWO & $\mathrm{NC}$ & -1.81 \\
\hline Yellow Warbler (Dendroica petechia)*** & 102 & 90 & 105 & LDM & OPWO & NC & -0.08 \\
\hline Chestnut-sided Warbler (Dendroica pensylvanica) ${ }^{* * *}$ & 0 & 0 & 2 & LDM & OPWO & NC & NA \\
\hline American Redstart (Setophaga ruticilla) $* * *$ & 13 & 10 & 20 & LDM & OPWO & $\mathrm{NC}$ & +0.48 \\
\hline Ovenbird (Seiurus aurocapillus) & 6 & 20 & 29 & LDM & WOOD & $\mathrm{NC}$ & +0.47 \\
\hline Northern Waterthrush (Seiurus noveboracensis) ${ }^{* * *}$ & 2 & 0 & 0 & LDM & WOOD & $\downarrow$ & NA \\
\hline Mourning Warbler (Oporornis philadelphia) ${ }^{* * *}$ & 0 & 1 & 1 & LDM & OPWO & NC & NA \\
\hline Common Yellowthroat (Geothlypis trichas) ${ }^{* * *}$ & 134 & 91 & 175 & LDM & WETL & NC & $-1.50 \downarrow$ \\
\hline Yellow-breasted Chat (Icteria virens) ${ }^{* *}$ & 6 & 6 & 11 & LDM & SHRU & NC & +1.76 \\
\hline Rose-breasted Grosbeak (Pheucticus ludovicianus)*** & 2 & 3 & 5 & LDM & OPWO & NC & +0.14 \\
\hline Black-headed Grosbeak (Pheucticus melanocephalus) ${ }^{* *}$ & 5 & 5 & 3 & LDM & OPWO & $\mathrm{NC}$ & +1.37 \\
\hline Blue Grosbeak (Guiraca caerulea) ${ }^{* *}$ & 1 & 1 & 0 & LDM & SHRU & NC & NA \\
\hline Lazuli Bunting (Passerina amoena) ${ }^{* *}$ & 8 & 12 & 17 & LDM & SHRU & $\mathrm{NC}$ & $+6.20 \uparrow \uparrow$ \\
\hline Indigo Bunting (Passerina cyanea) ${ }^{* * *}$ & 2 & 2 & 3 & LDM & OPWO & NC & $+3.92 \uparrow \uparrow$ \\
\hline Dickcissel (Spiza americana) ${ }^{* * *}$ & 63 & 34 & 14 & LDM & GRAS & NC & -2.64 \\
\hline Spotted Towhee (Pipilo maculatus) $)^{* *}$ & 94 & 83 & 118 & SDM & OPWO & NC & +0.43 \\
\hline Chipping Sparrow (Spizella passerina) ${ }^{* *}$ & 22 & 51 & 70 & LDM & OPWO & $\uparrow$ & $+5.29 \uparrow \uparrow \uparrow$ \\
\hline Clay-colored Sparrow (Spizella pallida) ${ }^{* * *}$ & 364 & 261 & 289 & LDM & SHRU & $\mathrm{NC}$ & $-3.51 \downarrow \downarrow$ \\
\hline Brewer's Sparrow (Spizella breweri) ${ }^{* * *}$ & 2 & 1 & 3 & LDM & SHRU & NC & +1.57 \\
\hline Field Sparrow (Spizella pusilla) ${ }^{* * *}$ & 49 & 65 & 74 & SDM & SHRU & NC & +3.58 \\
\hline Vesper Sparrow (Pooecetes gramineus) ${ }^{* * *}$ & 195 & 224 & 393 & SDM & GRAS & NC & $+2.49 \uparrow \uparrow \uparrow$ \\
\hline Lark Sparrow (Chondestes grammacus) ${ }^{* *}$ & 40 & 52 & 41 & LDM & OPTR & $\mathrm{NC}$ & +0.42 \\
\hline Lark Bunting (Calamospiza melanocorys)*** & 604 & 679 & 298 & LDM & GRAS & $\mathrm{NC}$ & $-3.40 \downarrow$ \\
\hline Savannah Sparrow (Passerculus sandwichensis) ${ }^{* *}$ & 516 & 134 & 276 & SDM & GRAS & $\downarrow$ & $-2.78 \downarrow$ \\
\hline Baird's Sparrow (Ammodramus bairdii) ${ }^{* *}$ & 170 & 77 & 125 & LDM & GRAS & NC & -2.75 \\
\hline Grasshopper Sparrow (Ammodramus savannarum) $)^{* * *}$ & 301 & 402 & 449 & LDM & GRAS & $\uparrow$ & $-5.24 \downarrow \downarrow \downarrow$ \\
\hline Le Conte's Sparrow (Ammodramus leconteii) ${ }^{* *}$ & 6 & 2 & 14 & SDM & GRAS & $\mathrm{NC}$ & $-5.02 \downarrow$ \\
\hline Nelson's Sharp-tailed Sparrow (Ammodramus nelsoni) ${ }^{* * *}$ & 3 & 3 & 13 & SDM & WETL & $\mathrm{NC}$ & +1.15 \\
\hline Song Sparrow (Melospiza melodia) ${ }^{* *}$ & 55 & 100 & 130 & SDM & SHRU & $\uparrow$ & -1.56 \\
\hline McCown's Longspur (Calcarius mccownii) ${ }^{* *}$ & 22 & 2 & 1 & SDM & GRAS & $\downarrow$ & -2.27 \\
\hline Chestnut-collared Longspur (Calcarius ornatus) ** & 1129 & 602 & 755 & SDM & GRAS & $\downarrow$ & -0.10 \\
\hline Bobolink (Dolichonyx oryzivorus) ${ }^{* * *}$ & 216 & 186 & 172 & LDM & GRAS & $\mathrm{NC}$ & -0.55 \\
\hline Red-winged Blackbird (Agelaius phoeniceus)** & 945 & 597 & 710 & SDM & WETL & $\downarrow$ & $-2.46 \downarrow \downarrow \downarrow$ \\
\hline Eastern Meadowlark (Sturnella magna)* & 0 & 0 & 1 & SDM & GRAS & $\mathrm{NC}$ & +1.75 \\
\hline Western Meadowlark (Sturnella neglecta) ${ }^{*}$ & 926 & 487 & 646 & SDM & GRAS & $\downarrow$ & +0.56 \\
\hline Yellow-headed Blackbird (Xanthocephalus xanthocephalus) ${ }^{* *}$ & 89 & 155 & 175 & LDM & WETL & $\mathrm{NC}$ & +0.65 \\
\hline Brewer's Blackbird (Euphagus cyanocephalus) ${ }^{* *}$ & 19 & 47 & 83 & SDM & SHRU & $\uparrow$ & $+6.49 \uparrow \uparrow$ \\
\hline Common Grackle (Quiscalus quiscula) ${ }^{* *}$ & 140 & 300 & 299 & SDM & OPTR & $\uparrow$ & $+4.71 \uparrow \uparrow \uparrow$ \\
\hline Brown-headed Cowbird (Molothrus ater) ${ }^{* *}$ & 460 & 643 & 610 & SDM & OPWO & $\uparrow$ & $+3.09 \uparrow \uparrow \uparrow$ \\
\hline
\end{tabular}


and semipermanent riparian areas); (7) Human-made Structures (human-made structures, fence rows and field borders, and road and railroad rights-of-way); and (8) Clay Buttes (portions of clay buttes that are mostly unvegetated).

\section{RESULTS}

Species composition. - One hundred and sixtyone breeding bird species (not including subspecies) were recorded within the 128 quartersections surveyed in at least one year, including 129 species in 1967, 144 in 1992, and 153 in 1993. These 161 species represent about $72 \%$ of the 223 species of North Dakota's breeding avifauna (Faanes and Stewart 1982). Most of the remaining species that were not observed on the quarter-sections are either rare, uncommon, localized, or irregular breeders in North Dakota (Faanes and Stewart 1982, DeSante and Pyle 1986).

The composition of breeding birds in North Dakota in 1992 and 1993 was similar to that in 1967 (Table 1). One hundred twenty-two species were recorded in all three years, 21 species were observed in two of the three years, and 18 species were detected in only one of the three years (Table 1). One species was recorded only in 1967, four species only in 1992, and 13 species only in 1993. Thirty-two species were observed only in 1992 and/or 1993, but not in 1967.

Most (92\%) of the 161 breeding bird species are migratory, and only a few species are considered permanent residents that show little or no seasonal movements in North Dakota (Tables 1 and 2). Of the species that migrate, 86 species are short-distance migrants and 62 are long-distance migrants. Moreover, migrants constituted over $96 \%$ of the total number of indicated pairs detected each year. Short-distance migrants composed over one-half of the observed indicated pairs each year.

Among breeding habitat associations, wetland species composed the largest proportion (32\%) of species, followed by species associated with open woodland or edge and grassland habitats (Table 1 and 2). In contrast, grassland birds composed the largest proportion of observed breeding pairs, accounting for $38 \%$ or more of the indicated pairs recorded in each year.

Frequencies of occurrence on sample units. - Sixty-nine species (i.e. common species) occurred in $10 \%$ or more of the sample quarter-sections in one or more years, including 48 species in 
TABLE 2. Composition of breeding birds and mean number of indicated pairs on 128 randomly selected quarter-sections in North Dakota, by year, breeding habitat, and migratory status.

\begin{tabular}{|c|c|c|c|c|c|c|c|}
\hline & \multirow{2}{*}{$\begin{array}{l}\text { No. of } \\
\text { species }\end{array}$} & \multicolumn{3}{|c|}{ Percent of observed pairs } & \multicolumn{3}{|c|}{ Mean pairs / 100 ha } \\
\hline & & 1967 & 1992 & 1993 & 1967 & 1992 & 1993 \\
\hline \multicolumn{8}{|c|}{ Breeding habitat } \\
\hline Wetland & 51 & 26.2 & 18.3 & 20.9 & 37.0 & 24.3 & 31.6 \\
\hline Grassland & 24 & 47.5 & 38.3 & 41.0 & 67.2 & 50.9 & 62.0 \\
\hline Shrubland & 14 & 6.3 & 7.2 & 7.5 & 8.9 & 9.6 & 11.3 \\
\hline Open habitat/scattered trees & 8 & 4.2 & 8.3 & 6.6 & 5.9 & 11.1 & 10.0 \\
\hline Open woodland/edge & 37 & 11.5 & 18.5 & 16.8 & 16.1 & 24.3 & 25.2 \\
\hline Woodland & 14 & 0.4 & 0.9 & 1.1 & 0.6 & 1.2 & 1.6 \\
\hline Residential/human structures & 8 & 3.1 & 7.2 & 5.3 & 4.4 & 9.5 & 8.0 \\
\hline Other & 5 & 0.8 & 1.3 & 0.8 & 1.1 & 1.7 & 1.2 \\
\hline \multicolumn{8}{|c|}{ Migratory status } \\
\hline Long-distance migrant & 62 & 31.2 & 38.6 & 31.7 & 43.8 & 51.1 & 47.7 \\
\hline Short-distance migrant & 86 & 67.4 & 57.8 & 65.0 & 95.4 & 76.8 & 98.3 \\
\hline Permanent resident & 13 & 1.4 & 3.6 & 3.3 & 2.0 & 4.7 & 4.9 \\
\hline
\end{tabular}

1967, 51 in 1992, and 64 in 1993 (Appendix 1). The increase in the number of common species between 1967 and 1992-93 was related primarily to the increase in statewide frequencies of some permanent residents and some species associated with human-made structures and woody vegetation (Table 1 , Appendix 1). In decreasing order, the five most frequently occurring species were Horned Lark (Eremophila alpestris), Brown-headed Cowbird, Western Meadowlark (Sturnella neglecta), Red-winged Blackbird, and Eastern Kingbird (Tyrannus tyrannus; Appendix 1). All but one (Downy Woodpecker [Picoides pubescens]) of these 69 common species occurred in all three years. Stewart and Kantrud (1972) commented that the absence of the Downy Woodpecker in 1967 was "somewhat surprising," given the species' widespread distribution in North Dakota.

Statewide population estimates. - The projected statewide population estimates for breeding birds in North Dakota were 25.5 million breeding pairs in 1967, 24.1 million in 1992, and 27.4 million in 1993. Projected statewide population estimates are given in Appendix 2 for the 69 species that had statewide frequencies of occurrence of $10 \%$ or higher in one or more years (i.e. common species). These 69 common species composed $95 \%$ of the projected statewide breeding bird populations in 1967, 92\% in 1992, and $92 \%$ in 1993 . By this criterion, more than onehalf (92 species) of the species observed in the three years were uncommon breeders. In decreasing order of abundance, the five most common species were the Horned Lark, Chestnut- collared Longspur (Calcarius ornatus), Redwinged Blackbird, Western Meadowlark, and Brown-headed Cowbird. Collectively, these species accounted for $31-41 \%$ of the estimated statewide breeding populations in the three years. The Horned Lark, a species that is most characteristic of cropland and heavily grazed grassland, accounted for over $10 \%$ of the projected statewide populations in each year.

Population changes. - Annual variation in breeding bird populations was considerable (Tables 2 and 3, Appendices 1 and 2). Annual breeding bird density for all species varied from 141 indicated breeding pairs per 100 ha in 1967 to 133 in 1992 and 151 in 1993. An evaluation of population changes for each species is beyond the scope of this paper. Rather, we restrict our evaluation to two broad patterns of population change evident in our data. First, most wetland species and many grassland species occurred less frequently and were less abundant during the dry year, 1992, than during the wetter years, 1967 and 1993. This pattern appears to be most pronounced and consistent for species associated with open water, such as waterfowl and the American Coot (Fulica americana). Second, many permanent residents and many species associated with human-made structures and woody vegetation generally increased in frequency and abundance between 1967 and 1992-93.

Of the 161 breeding bird species observed, $46(29 \%)$ had consistent and significant $(P \leq$ $0.10)$ population changes between 1967 and 1992 and between 1967 and 1993 (Table 1, Appendix 
TABLE 3. Land use changes on the 128 quarter-sections surveyed in 1967, 1992, and 1993, and percentage change between the two periods (1992 and 1993 averaged).

\begin{tabular}{lrrrr}
\hline \hline \multicolumn{1}{c}{ Habitat } & \multicolumn{3}{c}{ Area (ha) } & Percent change \\
\cline { 2 - 4 } \multicolumn{1}{c}{ Hropland } & 1967 & 1992 & 1993 & +6.0 \\
Grassland & 3,870 & 4,113 & 4,093 & -2.5 \\
Wetland & 2,142 & 2,093 & 2,084 & -27.0 \\
Hayland & 736 & 529 & 545 & -52.2 \\
Planted cover & 458 & 214 & 224 & +40.0 \\
Woodland/shrubland & 403 & 564 & 564 & +19.4 \\
Human-made structures & 268 & 320 & 320 & +13.6 \\
Clay buttes & 308 & 349 & 351 & +6.0 \\
\hline
\end{tabular}

2). Of these 46 species, 11 long-distance $\mathrm{mi}$ grants, 15 short-distance migrants, and 8 permanent residents exhibited increasing population changes, and 7 long-distance migrants, 5 short-distance migrants, and no permanent residents showed decreasing population changes. Twenty-four of the 34 (71\%) species with significantly increasing population changes were associated with woody vegetation or humanmade structures. In contrast, 11 of $12(92 \%)$ species with significantly decreasing population changes were associated with wetlands or grasslands. Grassland species, however, had equal numbers of significantly increasing (4) and decreasing (4) population changes.

Breeding Bird Survey trends. - Sample sizes were sufficient to estimate statewide BBS trends from 1967 to 1993 for 151 of the 161 observed species. Of the 64 species with statistically significant trends, 14 long-distance migrants, 15 short-distance migrants, and 7 permanent residents exhibited increasing trends. In contrast, 9 longdistance migrants, 18 short-distance migrants, and 1 permanent resident showed significantly decreasing trends. Twenty-eight of the $36(78 \%)$ species with significantly increasing trends were associated with human-made structures or woody vegetation. Most (23) of the 28 species with significantly decreasing trends were associated with wetlands or grasslands. Grassland species, however, had equal numbers of significantly increasing (5) and decreasing (5) population trends.

Land use changes. - Cropland and grassland were the dominant land uses in the study area, covering about three-quarters of the total area on the 128 quarter-sections in all three years (Table 3). The area of cropland increased by $2 \%$ between 1967 and 1992-93. In contrast, grassland, wetland, and hayland declined by about
$3 \%, 27 \%$, and 52\%, respectively, from their 1967 levels; all declines reflected nationwide trends (Samson and Knopf 1994, Dahl 1990, Herkert 1991). Woodland, human-made structures, and unvegetated portions of clay buttes increased by about $19 \%, 13 \%$, and $6 \%$, respectively, between 1967 and 1992-93. Planted cover increased by $40 \%$, which largely reflects the Conservation Reserve Program, a long-term, federal cropland retirement program that began in 1985 (Johnson and Igl 1995). Land use changes between 1992 and 1993 were relatively small.

\section{Discussion}

Species composition.-Compared with other ecological regions, the breeding avifauna of grasslands is relatively simple (Risser et al. 1981). A characteristic of grassland bird communities is low species diversity and numerical domination by a few species (Cody 1985). Although North Dakota is situated in the grassland biome of North America, the breeding avifauna of North Dakota is enriched by a diverse assemblage of birds with eastern, western, central, and northern North American affinities (Table 1; Stewart 1975). Nonetheless, the predominance of grassland birds is evident from our data. Collectively, eight species-Horned Lark, Western Meadowlark, Red-winged Blackbird, Brown-headed Cowbird, Lark Bunting (Calamospiza melanocorys), Savannah Sparrow (Passerculus sandwichensis), Grasshopper Sparrow (Ammodramus savannarum), and Chestnut-collared Longspur-accounted for 43-53\% of the breeding birds in North Dakota each year. All but two of these species (Brown-headed Cowbird and Red-winged Blackbird) are considered endemic or secondary grassland species in North America (Mengel 1970; Knopf 1988, 1994). 
Another characteristic of grassland bird assemblages in North America is the prevalence of short-distance migrants (MacArthur 1959, Willson 1976). In this study, short-distance migrants composed more than one-half of the observed species and indicated pairs in each year. These patterns are comparable to those of other grasslands in North America but contrast greatly with northeastern deciduous forests, where long-distance migrants and permanent residents dominate the breeding avifauna (MacArthur 1959, Willson 1976).

One species, Northern Waterthrush (Seiurus noveboracensis), was recorded only in 1967, compared with 32 species detected only in 1992 and/or 1993. Although differences in observer abilities between 1967 and 1992-93 may account for the increased number of species between the two periods (Table 1), many other recent or irregular events may have contributed to this increase. These include food-based nomadism (Pine Siskin [Carduelis pinus], DuBowy 1983; Short-eared Owl [Asio flammeus], Stewart 1975; Long-eared Owl [Asio otus], Marks et al. 1994), increased nest box availability (Hooded Merganser [Lophodytes cucullatus], Doty et al. 1984; Eastern Bluebird [Sialia sialis], Sauer and Droege 1990), successful reintroduction and management programs (Wild Turkey [Meleagris gallopavo], Johnson and Knue 1989; Canada Goose [Branta canadensis], Lee et al. 1984), and increased habitat availability (species associated with woody vegetation, see below). Nonetheless, most of the 32 species that were not observed in 1967 are considered rare, uncommon, localized, or irregular breeders in North Dakota (Faanes and Stewart 1982, DeSante and Pyle 1986).

Patterns of population changes.-Current evidence indicates that populations of some endemic grassland birds and many other species have declined in grassland regions in North America in recent decades (Knopf 1988, 1994; Finch 1992; Droege and Sauer 1994; USFWS 1995). Despite early indications of population declines in some breeding birds in the Midwest and Great Plains (Anonymous 1983, Robbins et al. 1986, Knopf 1988), birds in the midcontinent have not received the attention accorded other bird groups, such as Neotropical migrants in eastern forests (Hagan and Johnston 1992, Finch and Stangel 1993). In part, this may due to the inherent variability of bird populations in the Great Plains, making it difficult to detect long- term population changes. Species that breed in this region are believed to have evolved mechanisms in response to a naturally dynamic environment, including a high degree of behavioral plasticity in locating available habitat opportunistically (Cody 1985). Accordingly, many grassland and wetland birds undergo considerable annual changes in distribution and abundance in the Great Plains (Henny 1973, Trapp et al. 1981, Johnson and Grier 1988, Mulvihill 1989, Droege and Sauer 1989, Goosen et al. 1993, Igl and Johnson 1995).

Our results (Tables 1 and 2; Appendices 1 and 2) are consistent with earlier reports showing that breeding bird populations in grassland ecosystems are tremendously dynamic, exhibiting considerable annual variation in abundance and frequency of occurrence (Graber and Graber 1963, George et al. 1992, Zimmerman 1992). Within breeding habitat associations, our observed patterns of population change were remarkably similar and consistent among species with different migratory behaviors. First, many species associated with woody vegetation (e.g. House Wren [Troglodytes aedon]) and humanmade structures (e.g. Barn Swallow [Hirundo rustica]) generally increased between 1967 and 1992-93 (Appendices 1 and 2). Second, many grassland (e.g. Savannah Sparrow) and wetland (e.g. Wilson's Phalarope) species declined between 1967 and 1992-93, though many exhibited slight (e.g. American Coot), moderate (e.g. Western Meadowlark), or dramatic (e.g. Common Yellowthroat) increases between 1992 and 1993.

The two patterns of long-term population change evident in our data were consistent with trends from the BBS. For both surveys, most species with significantly increasing population changes were associated with human-made structures or woody vegetation, and most species with significantly decreasing population changes were associated with wetlands or grasslands. This concordance illustrates that both independently derived measures of population change likely were recording the same phenomena. Moreover, of the 46 and 64 species that had significant population changes in our survey and the BBS, respectively, 26 species had significant trends in both data sets; all 26 species were considered common species in North Dakota (Appendices 1 and 2). Only one of the 26 species, Grasshopper Sparrow, had a population change that was opposite in sign. This discrep- 
ancy could be attributed to many factors, including differences in survey methodology or trend analysis. However, the long-term trend from the BBS may have masked the recent population increase in Grasshopper Sparrows in the Great Plains; these increases have been associated with the increased availability of perennial grassland habitat established by the Conservation Reserve Program (Reynolds et al. 1994, Johnson and Igl 1995).

The causes of the above patterns may involve conditions in the breeding, migration, or wintering seasons (Sherry and Holmes 1992). The prevalence of parallel patterns of population change among habitat associations, but not among migratory behaviors, suggests that these population changes may be caused, at least in part, by conditions on the breeding grounds (Finch 1991). A common feature of breeding birds observed in this study is their dependence on habitats on the breeding grounds; most of these species breed in the northern Great Plains but winter elsewhere. For example, if breedingground conditions are primary influences on bird population dynamics, then populations of long-distance migrants should show similar patterns of change as short-distance migrants. The examples of Blue-winged Teal (Anas discors) and American Coot for wetlands, Baird's Sparrow (Ammodramus bairdii) and Savannah Sparrow for grasslands, and House Wren and Blue Jay for open woodlands are illustrative (Appendices 1 and 2).

What conditions or factors might limit populations on the breeding grounds in the northern Great Plains? Habitat loss and changes in land use have been viewed as major potential causes of population change for many breeding birds in the Great Plains (Knopf 1988, 1994. McNicholl 1988). Within the last century, the landscape of the Great Plains has been greatly modified by a number of human-induced changes. The once abundant grasslands and wetlands of the Great Plains have been drastically reduced, altered, and fragmented by intensive agriculture, roads, tree plantings, encroachment by woody vegetation, invasion of exotic plants, and other human activities. Knopf (1994) described several historic, contemporary, and continuing influences on breeding birds and their habitats in the Great Plains, including: (1) removal of native grazers and transformation to intensive, domestic livestock grazing; (2) cultivation of native grasslands; (3) loss of wetlands; and (4) woody development in the form of tree plantings and ecological invasions. The first three influences were implicated in the recent declines of grassland bird populations, as well as wetland associates. The fourth provided increased nesting opportunities for species associated with woody vegetation that generally were lacking or were limited in presettlement times. Likewise, Herkert (1994), Vickery et al. (1994), and Warner (1994) implicated fragmentation of grasslands in the recent declines of breeding birds in eastern and midwestern grasslands.

Before European settlement, the landscape of North Dakota included vast expanses of grassland, consisting primarily of northern mixedgrass prairie with some tallgrass prairie on the extreme eastern edge of the state (Risser et al. 1981). Moreover, the grasslands of the prairie pothole region (i.e. Drift Plains and the Missouri Coteau; Fig. 1) were dotted by millions of shallow wetland basins. Since settlement, North Dakota has lost about $49 \%$ of its wetlands (Dahl 1990 ) and $75 \%$ of its native grasslands (Samson and Knopf 1994), much of this before 1967. We compared the statewide changes in bird populations (Tables 1 and 2, Appendices 1 and 2) with the overall changes in land use area (Table 3) between 1967 and 1992-93. Long-term population changes in our study and BBS were consistent with the notion that these changes were, in part, caused by changes in land use on the breeding grounds (e.g. Knopf 1994). Declines in wetland species were commensurate with declines in wetland area. The increases in species associated with human-made structures and woody vegetation were consistent with the overall increases in area of those habitats. The declines in grassland birds paralleled the decrease in the combined total area of grassland and secondary grasslands (hayland and planted cover). Bernstein et al. (1990) repeated the historic survey of Kendeigh (1941) in northwestern Iowa and found similar declines in grassland birds and increases in species associated with woody vegetation.

Land use area, however, changed very little between 1992 and 1993, but our results suggest that many grassland and wetland species, as well as several other species, shifted similarly by increases in frequency and abundance between the two recent years (Tables 1 and 2, Appendices 1 and 2). Droege and Sauer (1989) and Peterjohn and Sauer (1993) suggested that breeding bird population declines on BBS routes in the late 1980s and increases in the early 1990s 
reflect extreme drought conditions and amelioration of drought conditions, respectively. Extended periods of below-average rainfall are known to affect populations of grassland and wetland birds (e.g. Johnson and Grier 1988). In this study, the increases between 1992 and 1993 corresponded with the amelioration of the longterm drought conditions and are consistent with observations of behavioral flexibility and opportunism in habitat selection (Cody 1985) and weather-related shifts in distribution and abundance by grassland (Wiens 1974) and wetland (Johnson and Grier 1988) species. George et al. (1992) also noted rapid recoveries of grassland bird densities after drought. Reproductive success of grassland birds varies annually, with very poor productivity during some drought years (George et al. 1992).

In conclusion, our results indicate that BBS data reflect real population changes for many common species in North Dakota. The increase in species associated with human-made structures and woody vegetation and decreases in wetland and grassland species in agricultural landscapes have been reported previously, both in North America (McNicholl 1988, Knopf 1994, Herkert 1995) and elsewhere (Böhning-Gause and Bauer 1996). Our data suggest that land use changes, and probably drought conditions, influenced some breeding bird population changes in North Dakota between 1967 and 1992-93. Although these factors would not necessarily affect different species to the same extent, the fact that our surveys detected significant population changes for several common species (e.g. Northern Shoveler [Anas clypeata]) that the BBS did not detect and vice versa (e.g. Sora [Porzana carolina]) deserves further investigation. Furthermore, we cannot attribute all population changes to conditions on the breeding grounds. However, because many birds in the Great Plains are short-distance migrants, many of the factors driving population changes on the Great Plains are likely North American processes (Knopf 1994, Herkert 1995). Nonetheless, our data indicate that factors on the breeding grounds are having a major effect on breeding bird populations in the northern Great Plains.

\section{ACKNOWLEDGMENTS}

We are grateful to $H$. A. Kantrud, National Biological Service, for his advice and guidance throughout all phases of this study. J. R. Sauer of the National Biological Service and B. G. Peterjohn of the U. S. Fish and Wildlife Service supplied trends from the Breeding Bird Survey. This study would not have been possible without the cooperation of the many land owners, operators, and managers who allowed us to survey birds on their land. We thank C. J. Johnson and M. D. Schwartz for assistance in the field. R. L. Hutto, H. A. Kantrud, T. E. Martin, C. S. Robbins, P. F. Springer, and three anonymous reviewers commented on earlier drafts of this manuscript. This study was inspired by the innovative thoughts and foresight of Robert E. Stewart (1913-1993), whose scientific accomplishments were innumerable; we dedicate this paper to his memory.

\section{LITERATURE Cited}

AMERICAN ORNITHOLOGISTs' UNION. 1983. Check-list of North American birds, 6th ed. American Ornithologists' Union, Washington, D.C.

ANONYMOUs. 1983. The declining grassland birds. Illinois Natural History Survey Report No. 227.

Askins, R. A. 1993. Population trends in grassland, shrubland, and forest birds in eastern North America. Current Ornithology 11:1-34.

Askins, R. A., J. F. LYNCH, AND R. GREENBERG. 1990. Population declines in migratory birds in eastern North America. Current Ornithology 7:1-57.

BERNSTEIN, N. P., K. K. BAKER, AND S. R. WILMOT. 1990. Changes in a prairie bird population from 1940 to 1989. Journal of the Iowa Academy of Science 97:115-120.

BöHNING-GAUSE, K., AND H.-G. BAUER. 1996. Changes in species abundance, distribution, and diversity in a central European bird community. Conservation Biology 10:175-187.

Box, G. E. P., AND G. C. TIAo. 1973. Bayesian inference in statistical analysis. Addison-Wesley, Reading, Massachusetts.

Cochran, W. G. 1977. Sampling techniques, 3rd ed. John Wiley and Sons, New York.

CoDY, M. L. 1985. Habitat selection in grassland and open-country birds. Pages 191-226 in Habitat selection in birds (M. L. Cody, Ed.). Academic Press, Orlando, Florida.

DAHL, T. E. 1990. Wetland losses in the United States, 1780 's to 1980 's. U.S. Fish and Wildlife Service, Washington, D.C.

Desante, D., and P. Pyle. 1986. Distributional checklist of North American birds. Volume 1: United States and Canada. Artemisia Press, Lee Vining, California.

Doty, H. A., F. B. LeE, A. D. KRuse, J. W. Mathews, AND P. M. ARNOLD. 1984. Wood Duck and Hooded Merganser nesting on Arrowwood NWR, North Dakota. Journal of Wildlife Management 48:577-580.

Droege, S., AND J. R. SAUeR. 1989. North American Breeding Bird Survey Annual summary 1988. U.S. 
Fish and Wildlife Service Biological Report 89(13), Washington, D.C.

Droege, S., AND J. R. SAUER. 1994. Are more North American species decreasing than increasing? Pages 297-306 in Bird numbers 1992. Distribution, monitoring and ecological aspects (E. J. M. Hagemeijer and T. J. Verstrael, Eds.). Proceedings of the 12th International Conference of IBCC and EOAC, Noordwijkerhout, The Netherlands.

DuBowy, P. J. 1983. Additional records of passerines feeding on poplar galls, and a possible mechanism for summer nomadism in boreal finches. Prairie Naturalist 15:63-64.

DuNN, E. H., AND D. J. T. Hussell. 1995. Using migration counts to monitor landbird populations: Review and evaluation of current status. Current Ornithology 12:43-88.

EHRLICH, P. R., D. S. DOBKIN, AND D. WhEYE. 1988. The birder's handbook. Simon and Schuster, New York.

FAANES, C. A., AND R. E. Stewart. 1982. Revised checklist of North Dakota birds. Prairie Naturalist 14:81-92.

FiNCH, D. M. 1991. Population ecology, habitat requirements, and conservation of Neotropical migratory birds. U.S. Forest Service General Technical Report RM-205, Fort Collins, Colorado.

FiNCH, D. M. 1992. Threatened, endangered, and vulnerable species of terrestrial vertebrates in the Rocky Mountain region. U.S. Forest Service General Technical Report RM-215, Fort Collins, Colorado.

FinCH, D. M., AND P. W. STANGel (Eds.). 1993. Status and management of Neotropical migratory birds. U.S. Forest Service General Technical Report RM229, Fort Collins, Colorado.

FretWELl, S. D., AND J. S. CALVER. 1970. On territorial behavior and other factors influencing habitat distribution in birds. II. Sex ratio variation in the Dickcissel. Acta Biotheoretica 19:37-44.

GeIssLeR, P. H. AND J. R. SAUER. 1990. Topics in routeregression analysis. Pages 54-57 in Survey designs and statistical methods for the estimation of avian population trends (J. R. Sauer and S. Droege, Eds.). U.S. Fish and Wildlife Service Biological Report 90(1), Washington, D.C.

GeORGE, T. L., A. C. FowLER, R. L. KNIGHT, AND L. C. MCEWEN. 1992. Impacts of a severe drought on grassland birds in western North Dakota. Ecological Applications 2:275-284.

GibBS, J. P., AND J. FAABORG. 1990. Estimating the viability of Ovenbird and Kentucky Warbler populations in forest fragments. Conservation Biology 4:193-196.

Goosen, J. P., S. Brechtel, K. D. De SMet, D. HJertaAs, AND C. WERSCHLER. 1993. Canadian Baird's Sparrow recovery plan. Recovery of Nationally Endangered Wildlife Report No. 3, Canadian Wildlife Federation, Ottawa.
GRABER, R. R., AND J. W. GRABER. 1963. A comparative study of bird populations in Illinois, 1906-1909 and 1956-1958. Illinois Natural History Survey Bulletin 28:383-528.

HaGAN, J. M., III. 1993. Decline of the Rufous-sided Towhee in the eastern United States. Auk 110: 863-874.

HaGaN, J. M., III, AND D. W. Johnston (Eds.). 1992 Ecology and conservation of Neotropical migrant landbirds. Smithsonian Institution Press, Washington, D.C.

HAMMOND, M. C. 1969. Notes on conducting waterfowl breeding population surveys in the north central states. Pages 238-254 in Saskatoon Wetlands Seminar, Canadian Wildlife Service Report Series No. 6.

HARRISON, P. 1983. Seabirds: An identification guide. Houghton Mifflin, Boston, Massachusetts.

Hayman, P., J. Marchant, AND T. Prater. 1986. Shorebirds: An identification guide. Houghton Mifflin, Boston, Massachusetts

HENNY, C. J. 1973. Drought displaced movement of North American pintails into Siberia. Journal of Wildlife Management 37:23-29.

Herkert, J. R. 1991. Prairie birds of Illinois: Population response to two centuries of habitat change. Illinois Natural History Survey Bulletin 34:393399.

HERKERT, J, R. 1994. The effects of habitat fragmentation on midwestern grassland bird communities. Ecological Applications 4:461-471.

HERKERT, J. R. 1995. An analysis of midwestern breeding bird population trends: 1966-1993. American Midland Naturalist 134:41-50.

IGL, L. D., AND D. H. JoHnson. 1995. Dramatic increase of Le Conte's Sparrow in Conservation Reserve Program fields in the northern Great Plains. Prairie Naturalist 27:89-94.

JENSEN, R. E. 1972. Climate of North Dakota. U.S. National Weather Service, Fargo, North Dakota.

JoHNSON, D. H. 1977. Some Bayesian statistical techniques useful in estimating frequency and density. U.S. Fish and Wildlife Service Special Scientific Report, Wildlife No. 203, Washington, D.C.

JOHNSON, D. H., AND J. W. GRIER. 1988. Determinants of breeding distributions of ducks. Wildlife Monographs 100:1-37.

JoHNSON, D. H., AND L. D. IGL. 1995. Contributions of the Conservation Reserve Program to populations of breeding birds in North Dakota. Wilson Bulletin 107:709-718.

Johnson, D. H., AND M. D. Schwartz. 1993. The Conservation Reserve Program and grassland birds. Conservation Biology 7:934-937.

Johnson, M. D., AND J. KNUE. 1989. Feathers from the prairie: A short history of upland game birds, 2nd ed. North Dakota Game and Fish Department, Bismarck.

Johnston, D. W., AND J. M. HaGaN, III. 1992. An 
analysis of long-term breeding bird censuses from eastern deciduous forests. Pages 75-84 in Ecology and conservation of Neotropical migrant landbirds (J. M. Hagan, III and D. W. Johnston, Eds.). Smithsonian Institution Press, Washington, D.C.

KANTRUD, H. A. 1982. Maps of distribution and abundance of selected species of birds on uncultivated native upland grasslands and shrubsteppe in the northern Great Plains. U.S. Fish and Wildlife Service, FWS/OBS-82/31.

KENDEIGH, S. C. 1941. Birds of a prairie community. Condor 43:165-174.

KNOPF, F. L. 1988. Conservation of steppe birds in North America. Pages 27-41 in Ecology and conservation of grassland birds (P. D. Goriup, Ed.). International Council for Bird Preservation Technical Publication No. 7.

KNOPF, F. L. 1994. Avian assemblages on altered grasslands. Studies in Avian Biology 15:247-257.

LeE, F. B., C. H. SChroeder, T. L. KuCK, L. J. SCHOONOVER, M. A. JohnSON, H. K. Nelson, AND C. A. BeAUdUY. 1984. Rearing and restoring giant Canada Geese in the Dakotas. North Dakota Game and Fish Department, Bismarck.

MACARTHUR, R. H. 1959. On the breeding distribution pattern of North American migrant birds. Auk 76:318-325.

MADGE, S., AND H. BURN. 1988. Waterfowl: An identification guide to the ducks, geese and swans of the world. Houghton Mifflin, Boston, Massachusetts.

MARKS, J. S., D. L. EvaNS, AND D. W. Holt. 1994. Long-eared Owl (Asio otus). In The birds of North America, no. 133 (A. Poole and F. Gill, Eds.) Academy of Natural Sciences, Philadelphia, and American Ornithologists' Union, Washington, D.C.

MCNicholl, M. K. 1988. Ecological and human influences on Canadian populations of grassland birds. Pages 1-25 in Ecology and conservation of grassland birds (P. D. Goriup, Ed.). International Council for Bird Preservation Technical Publication No. 7.

Mengel, R. M. 1970. The North American central plains as an isolating agent in bird speciation. Pages 280-340 in Pleistocene and recent environments of the central Great Plains (W. Dort and J. K. Jones, Eds.). University of Kansas Press, Lawrence.

Microlmages, INC. 1992. A guide to map and image processing. MicroImages Press, Lincoln, Nebraska.

MulviHILL, R. S. 1989. The occurrence of Dickcissels (Spiza americana) in western Pennsylvania during the 1988 nesting season: Its possible bearing on the unusual history in eastern North America. Pennsylvania Birds 2:83-87.

NATIONAL OCEANIC AND ATMOSPHERIC ADMINISTRATION. 1967, 1988-1993. Climatological data:
North Dakota. National Climatic Data Center, Asheville, North Carolina.

Nelms, C. O., W. J. Bleier, D. L. Otis, AND G. M. Linz. 1994. Population estimates of breeding blackbirds in North Dakota, 1967, 1981-1982 and 1990. American Midland Naturalist 132:256-263.

Peterjohn, B. G., AND J. R. Sauer. 1993. North American Breeding Bird Survey annual summary, 19901991. Bird Populations 1:52-67.

RAPPOLE, J. H., E. S. MORTON, T. E. LoveJOY, III, AND J. L. Ruos. 1983. Nearctic avian migrants in the Neotropics. U.S. Fish and Wildlife Service, Washington, D.C.

REYNOLDS, R. E., T. L. SHAFFER, J. R. SAUER, AND B. G. PETERJOHN. 1994. Conservation Reserve Program: Benefit for grassland birds in the Northern Plains. Transactions of the North American Wildlife and Natural Resources Conference 59:328336.

RISSER, P. G., E. C. BIRNEY, H. D. BLOCKER, S. W. MAY, W. J. Parton, AND J. A. Wiens. 1981. The true prairie ecosystem. Hutchinson Ross Publishing, Stroudsburg, Pennsylvania.

RobBins, C. S., D. BystraK, AND P. H. GeIsSLER. 1986. The Breeding Bird Survey: Its first fifteen years, 1965-1979. U.S. Fish and Wildlife Service Resource Publication No. 157, Washington, D.C.

RobBiNs, C. S., S. DROEGE, AND J. R. SAUER. 1989. Monitoring bird populations with Breeding Bird Survey and atlas data. Annales Zoologici Fennici 26:297-304.

SAMSON, F., AND F. KNOPF. 1994. Prairie conservation in North America. BioScience 44:418-421.

SAUER, J. R., AND S. DROEGE. 1990. Recent population trends of the Eastern Bluebird. Wilson Bulletin 102:239-252.

SHERRY, T. W., AND R. T. Holmes. 1992. Are populations of Neotropical migrant birds limited in summer or winter? Implications for management. Pages 47-57 in Status and management of Neotropical migratory birds (D. M. Finch and P. W. Stangel, Eds.). U.S. Forest Service General Technical Report RM-229, Fort Collins, Colorado.

SPEIRS, J. M., AND R. ORENSTEIN. 1967. Bird populations in fields of Ontario County, 1965. Canadian Field-Naturalist 81:175-183.

STEWART, R. E. 1975. Breeding birds of North Dakota. Tri-College Center for Environmental Studies, Fargo, North Dakota.

StEWART, R. E., AND H. A. Kantrud. 1971. Classification of natural ponds and lakes in the glaciated prairie region. U.S. Fish and Wildlife Service Resource Publication No. 92, Washington, D.C.

Stewart, R. E., AND H. A. Kantrud. 1972. Population estimates of breeding birds in North Dakota. Auk 89:766-788.

TEMPLE, S. A., AND J. R. CARY. 1990. Using checklist records to reveal trends in bird populations. Pages 98-104 in Survey designs and statistical methods 
for the estimation of avian population trends (J. R. Sauer and S. Droege, Eds.). U.S. Fish and Wildlife Service Biological Report 90(1), Washington, D.C.

Thompson, F. R., S. J. LeWIS, J. GREEN, AND D. EWERT. 1993. Status of Neotropical migrant landbirds in the Midwest: Identifying species of management concern. Pages 145-158 in Status and management of Neotropical migratory birds (D. M. Finch and P. W. Stangel, Eds.). U.S. Forest Service General Technical Report RM-229, Fort Collins, Colorado.

Trapp, J. L., M. A. Robus, G. J. TANS, AND M. M. TANs. 1981. First breeding record of the Sora and American Coot in Alaska: With comments on drought displacement. American Birds 35:901902.

U.S. FISH AND Wildlife SERvice. 1995. Migratory nongame birds of management concern in the United States: The 1995 list. U.S. Fish and Wildlife Service, Washington, D.C.
Vickery, P. D., M. L. Hunter, JR., AND S. M. Melvin. 1994. Effects of habitat area on the distribution of grassland birds in Maine. Conservation Biology 8:1087-1097.

WARNER, R. E. 1994. Agricultural land use and grassland habitat in Illinois: Future shock for midwestern birds? Conservation Biology 8:147-156.

WIENS, J. A. 1974. Climatic instability and the "ecological saturation" of bird communities in North American grasslands. Condor 76:385-400.

WILlson, M. F. 1976. The breeding distribution of North American migrant birds: A critique of MacArthur (1959). Wilson Bulletin 88:582-587.

ZIMMERMAN, J. L. 1992. Density-independent factors affecting the avian diversity of the tallgrass prairie community. Wilson Bulletin 104:85-94.

Associate Editor: R. L. Hutto

APPENDIX 1. Statewide frequencies of occurrence (confidence interval) of common bird species (i.e. species with frequencies greater than 10\%) in North Dakota.

\begin{tabular}{|c|c|c|c|}
\hline \multirow[b]{2}{*}{ Species } & \multicolumn{3}{|c|}{ Year } \\
\hline & 1967 & 1992 & 1993 \\
\hline Green-winged Teal & $18.0(11.8-24.9)$ & $3.1(0.8-6.5)$ & $11.7(6.6-17.8)$ \\
\hline Mallard & $60.9(52.3-69.3)$ & $35.9(27.9-44.3)$ & $53.9(45.3-62.4)$ \\
\hline Northern Pintail & $52.3(43.7-60.9)$ & $13.3(7.9-19.6)$ & $24.2(17.1-32.0)$ \\
\hline Blue-winged Teal & $38.3(29.9-47.0)$ & $18.8(12.4-25.9)$ & $25.0(17.6-33.1)$ \\
\hline Northern Shoveler & $28.1(20.6-36.2)$ & $9.4(4.9-14.8)$ & $17.2(11.2-23.9)$ \\
\hline Gadwall & $28.1(20.6-36.2)$ & $14.1(8.5-20.5)$ & $25.8(18.5-33.7)$ \\
\hline American Wigeon & $11.7(6.6-17.8)$ & $7.0(3.2-11.9)$ & $7.8(3.9-12.6)$ \\
\hline Northern Harrier & $11.7(6.6-17.8)$ & $16.4(10.5-23.1)$ & $25.0(17.6-33.1)$ \\
\hline Swainson's Hawk & $5.5(2.1-10.0)$ & $10.9(6.1-16.7)$ & $19.5(13.1-26.7)$ \\
\hline Red-tailed Hawk & $5.5(2.1-10.0)$ & $8.6(4.2-14.1)$ & $12.5(7.3-18.6)$ \\
\hline Gray Partridge & $11.7(6.6-17.8)$ & $20.3(13.9-27.4)$ & $20.3(13.9-27.4)$ \\
\hline Ring-necked Pheasant & $2.3(0.4-5.5)$ & $11.7(6.6-17.8)$ & $17.2(11.2-23.9)$ \\
\hline Sharp-tailed Grouse & $7.8(3.9-12.6)$ & $12.5(7.3-18.6)$ & $11.7(6.6-17.8)$ \\
\hline Sora & $13.3(7.9-19.6)$ & $7.8(3.9-12.6)$ & $23.4(16.3-31.3)$ \\
\hline American Coot & $23.4(16.3-31.3)$ & $6.3(2.7-10.8)$ & $14.1(8.5-20.5)$ \\
\hline Killdeer & $49.2(40.7-57.8)$ & $43.0(34.6-51.5)$ & $56.3(47.6-64.7)$ \\
\hline Willet & $13.3(7.9-19.6)$ & $7.8(3.9-12.6)$ & $8.6(4.2-14.1)$ \\
\hline Upland Sandpiper & $28.1(20.6-36.2)$ & $38.3(29.9-47.0)$ & $37.5(29.3-46.0)$ \\
\hline Marbled Godwit & $11.7(6.6-17.8)$ & $5.5(2.1-10.0)$ & $9.4(4.9-14.8)$ \\
\hline Wilson's Phalarope & $22.7(15.7-30.3)$ & $6.3(2.7-10.8)$ & $10.2(5.5-15.8)$ \\
\hline Black Tern & $21.1(14.4-28.5)$ & $3.9(1.1-8.1)$ & $10.2(5.5-15.8)$ \\
\hline Mourning Dove & $54.7(46.1-63.2)$ & $53.9(45.3-62.4)$ & $60.2(51.6-68.5)$ \\
\hline Common Nighthawk & $5.5(2.1-10.0)$ & $7.0(3.2-11.9)$ & $10.2(5.5-15.8)$ \\
\hline Black-billed Cuckoo & $4.7(1.6-9.0)$ & $16.4(10.5-23.1)$ & $7.0(3.2-11.9)$ \\
\hline Downy Woodpecker & 0 & $6.3(2.7-10.8)$ & $10.9(6.1-16.7)$ \\
\hline Yellow-shafted Flicker & $16.4(10.5-23.1)$ & $14.1(8.5-20.5)$ & $18.8(12.4-25.9)$ \\
\hline Willow Flycatcher & $12.5(7.3-18.6)$ & $5.5(2.1-10.0)$ & $19.5(13.1-26.7)$ \\
\hline Least Flycatcher & $7.0(3.2-11.9)$ & $11.7(6.6-17.8)$ & $15.6(9.8-22.3)$ \\
\hline Say's Phoebe & $3.1(0.8-6.5)$ & $3.1(0.8-6.5)^{\prime}$ & $12.5(7.3-18.6)$ \\
\hline Western Kingbird & $38.3(29.9-47.0)$ & $64.1(55.7-72.1)$ & $60.9(52.3-69.3)$ \\
\hline Eastern Kingbird & $54.7(46.1-63.2)$ & $76.6(68.7-83.7)$ & $74.2(66.3-81.5)$ \\
\hline Horned Lark & $85.9(79.5-91.5)$ & $86.7(80.4-92.1)$ & $85.2(78.5-90.9)$ \\
\hline
\end{tabular}


APPENDIX 1. Continued.

\begin{tabular}{|c|c|c|c|}
\hline \multirow[b]{2}{*}{ Species } & \multicolumn{3}{|c|}{ Year } \\
\hline & 1967 & 1992 & 1993 \\
\hline Bank Swallow & $7.8(3.9-12.6)$ & $10.2(5.5-15.8)$ & $12.5(7.3-18.6)$ \\
\hline Cliff Swallow & $5.5(2.1-10.0)$ & $14.8(9.1-21.5)$ & $18.0(11.8-24.9)$ \\
\hline Barn Swallow & $39.8(31.5-48.4)$ & $57.0(48.5-65.4)$ & $58.6(50.1-66.9)$ \\
\hline Blue Jay & $7.0(3.2-11.9)$ & $11.7(6.6-17.8)$ & $10.9(6.1-16.7)$ \\
\hline American Crow & $18.8(12.4-25.9)$ & $9.4(4.9-14.8)$ & $19.5(13.1-26.7)$ \\
\hline Black-capped Chickadee & $2.3(0.4-5.5)$ & $12.5(7.3-18.6)$ & $15.6(9.8-22.3)$ \\
\hline House Wren & $15.6(9.8-22.3)$ & $35.9(27.9-44.3)$ & $32.8(25.1-40.9)$ \\
\hline Marsh Wren & $6.3(2.7-10.8)$ & $9.4(4.9-14.8)$ & $12.5(7.3-18.6)$ \\
\hline American Robin & $15.6(9.8-22.3)$ & $33.6(25.7-41.9)$ & $35.9(27.9-44.3)$ \\
\hline Gray Catbird & $10.9(6.1-16.7)$ & $14.8(9.1-21.5)$ & $14.1(8.5-20.5)$ \\
\hline Brown Thrasher & $18.8(12.4-25.9)$ & $34.4(26.5-42.6)$ & $41.4(33.1-49.9)$ \\
\hline Cedar Waxwing & $7.0(3.2-11.9)$ & $14.1(8.5-20.5)$ & $10.2(5.5-15.8)$ \\
\hline Loggerhead Shrike & $6.3(2.7-10.8)$ & $10.9(6.1-16.7)$ & $11.7(6.6-17.8)$ \\
\hline Warbling Vireo & $6.3(2.7-10.8)$ & $9.4(4.9-14.8)$ & $14.8(9.1-21.5)$ \\
\hline Yellow Warbler & $22.7(15.7-30.3)$ & $19.5(13.1-26.7)$ & $21.1(14.4-28.5)$ \\
\hline Common Yellowthroat & $29.7(22.1-37.8)$ & $28.9(21.5-36.8)$ & $39.1(30.7-47.7)$ \\
\hline Chipping Sparrow & $5.5(2.1-10.0)$ & $14.8(9.1-21.5)$ & $14.8(9.1-21.5)$ \\
\hline Clay-colored Sparrow & $39.8(31.5-48.4)$ & $35.9(27.9-44.3)$ & $35.2(27.1-43.6)$ \\
\hline Vesper Sparrow & $52.3(43.7-60.9)$ & $50.0(41.4-58.6)$ & $52.3(43.7-60.9)$ \\
\hline Lark Sparrow & $8.6(4.2-14.1)$ & $11.7(6.6-17.8)$ & $9.4(4.9-14.8)$ \\
\hline Lark Bunting & $41.4(33.1-49.9)$ & $40.6(32.1-49.4)$ & $25.0(17.6-33.1)$ \\
\hline Savannah Sparrow & $63.3(54.7-71.5)$ & $28.1(20.6-36.2)$ & $44.5(36.1-53.1)$ \\
\hline Baird's Sparrow & $24.2(17.1-32.0)$ & $18.0(11.8-24.9)$ & $20.3(13.9-27.4)$ \\
\hline Grasshopper Sparrow & $48.4(39.9-57.0)$ & $50.0(41.4-58.6)$ & $46.1(37.6-54.7)$ \\
\hline Song Sparrow & $20.3(13.9-27.4)$ & $25.8(18.5-33.7)$ & $32.8(25.1-40.9)$ \\
\hline Chestnut-collared Longspur & $47.7(39.1-56.3)$ & $39.1(30.7-47.7)$ & $39.8(31.5-48.4)$ \\
\hline Bobolink & $44.5(36.1-53.1)$ & $36.7(28.5-45.3)$ & $43.8(35.3-52.4)$ \\
\hline Red-winged Blackbird & $80.5(73.3-86.9)$ & $67.2(59.1-74.9)$ & $67.2(59.1-74.9)$ \\
\hline Western Meadowlark & $94.5(90.0-97.9)$ & $77.3(69.7-84.3)$ & $82.0(75.1-88.2)$ \\
\hline Yellow-headed Blackbird & $11.7(6.6-17.8)$ & $16.4(10.5-23.1)$ & $18.0(11.8-24.9)$ \\
\hline Brewer's Blackbird & $7.8(3.9-12.6)$ & $9.4(4.9-14.8)$ & $17.2(11.2-23.9)$ \\
\hline Common Grackle & $25.8(18.5-33.7)$ & $39.1(30.7-47.7)$ & $34.4(26.5-42.6)$ \\
\hline Brown-headed Cowbird & $81.3(74.1-87.6)$ & $83.6(76.9-89.5)$ & $89.1(83.3-93.9)$ \\
\hline Orchard Oriole & $9.4(4.9-14.8)$ & $18.0(11.8-24.9)$ & $18.8(12.4-25.9)$ \\
\hline Baltimore Oriole & $11.7(6.6-17.8)$ & $21.1(14.4-28.5)$ & $14.8(9.1-21.5)$ \\
\hline American Goldfinch & $38.3(29.9-47.0)$ & $34.4(26.5-42.6)$ & $43.0(34.6-51.5)$ \\
\hline House Sparrow & $17.2(11.2-23.9)$ & $21.9(14.9-29.6)$ & $22.7(15.7-30.3)$ \\
\hline
\end{tabular}

APPENDIX 2. Statewide breeding population estimates $(1,000$ s of pairs, with confidence intervals $)$ of common species of birds (i.e. species with statewide frequencies greater than 10\%) in North Dakota.

\begin{tabular}{lccc}
\hline \hline & & Year & \\
\cline { 2 - 4 } \multicolumn{1}{c}{ Species } & 1967 & 1992 & 1993 \\
\hline Green-winged Teal & $96(54-139)$ & $24(0-47)$ & $96(30-161)$ \\
Mallard & $457(373-541)$ & $249(148-349)$ & $439(294-585)$ \\
Northern Pintail & $371(295-448)$ & $50(26-75)$ & $127(79-176)$ \\
Blue-winged Teal & $612(425-799)$ & $216(113-319)$ & $312(177-448)$ \\
Northern Shoveler & $189(108-269)$ & $55(20-90)$ & $112(55-170)$ \\
Gadwall & $208(101-315)$ & $246(55-437)$ & $259(126-393)$ \\
American Wigeon & $53(20-87)$ & $49(0-102)$ & $71(0-132)$ \\
Northern Harrier & $33(17-49)$ & $46(28-64)$ & $75(53-98)$ \\
Swainson's Hawk & $16(5-27)$ & $31(16-47)$ & $55(35-75)$ \\
Red-tailed Hawk & $15(4-25)$ & $24(10-38)$ & $34(19-49)$ \\
Gray Partridge & $38(19-56)$ & $89(49-128)$ & $79(47-112)$ \\
Ring-necked Pheasant & $7(0-13)$ & $53(24-83)$ & $114(49-179)$ \\
\hline
\end{tabular}


APPENDIX 2. Continued.

\begin{tabular}{|c|c|c|c|}
\hline \multirow[b]{2}{*}{ Species } & \multicolumn{3}{|c|}{ Year } \\
\hline & 1967 & 1992 & 1993 \\
\hline Sharp-tailed Grouse & $39(12-67)$ & $102(23-182)$ & $72(32-112)$ \\
\hline Sora & $68(34-102)$ & $90(0-173)$ & $167(100-235)$ \\
\hline American Coot & $761(206-1,315)$ & $169(0-414)$ & $271(37-504)$ \\
\hline Killdeer & $227(176-277)$ & $248(178-318)$ & $312(226-397)$ \\
\hline Willet & $39(22-56)$ & $35(10-61)$ & $60(12-108)$ \\
\hline Upland Sandpiper & $139(93-185)$ & $236(166-307)$ & $198(138-258)$ \\
\hline Marbled Godwit & $37(19-55)$ & $18(5-31)$ & $31(14-49)$ \\
\hline Wilson's Phalarope & $157(97-218)$ & $66(8-125)$ & $79(31-128)$ \\
\hline Black Tern & $254(101-408)$ & $86(0-192)$ & $83(28-139)$ \\
\hline Mourning Dove & $628(478-777)$ & $742(538-945)$ & $733(542-923)$ \\
\hline Common Nighthawk & $23(5-40)$ & $27(8-45)$ & $35(15-54)$ \\
\hline Black-billed Cuckoo & $15(3-27)$ & $65(36-94)$ & $22(8-36)$ \\
\hline Downy Woodpecker & 0 & $19(6-33)$ & $45(19-71)$ \\
\hline Yellow-shafted Flicker & $87(45-128)$ & $55(28-81)$ & $76(43-110)$ \\
\hline Willow Flycatcher & $63(27-98)$ & $37(6-69)$ & $79(46-113)$ \\
\hline Least Flycatcher & $48(12-85)$ & $112(35-189)$ & $169(77-260)$ \\
\hline Western Kingbird & $221(160-282)$ & $427(342-512)$ & $390(291-488)$ \\
\hline Eastern Kingbird & $360(276-444)$ & $706(586-827)$ & $536(432-641)$ \\
\hline Say's Phoebe & $12(1-24)$ & $8(1-16)$ & $37(20-54)$ \\
\hline Horned Lark & $2,772(2,407-3,136)$ & $2,412(2,082-2,742)$ & $3,672(3,164-4,179)$ \\
\hline Bank Swallow & $161(0-300)$ & $246(0-473)$ & $131(48-213)$ \\
\hline Cliff Swallow & $308(0-629)$ & $743(173-1,313)$ & $477(111-842)$ \\
\hline Barn Swallow & $206(155-257)$ & $408(305-510)$ & $420(323-516)$ \\
\hline Blue Jay & $29(7-51)$ & $58(25-92)$ & $66(26-107)$ \\
\hline American Crow & $60(37-83)$ & $28(13-42)$ & $58(38-78)$ \\
\hline Black-capped Chickadee & $6(0-12)$ & $59(26-93)$ & $66(38-94)$ \\
\hline House Wren & $111(52-170)$ & $473(306-641)$ & $449(287-611)$ \\
\hline Marsh Wren & $112(0-218)$ & $250(0-526)$ & $336(0-649)$ \\
\hline American Robin & $144(66-221)$ & $254(163-345)$ & $267(167-367)$ \\
\hline Gray Catbird & $103(38-168)$ & $94(47-142)$ & $82(37-126)$ \\
\hline Brown Thrasher & $93(48-138)$ & $187(130-244)$ & $229(165-293)$ \\
\hline Cedar Waxwing & $49(8-89)$ & $279(68-489)$ & $90(28-152)$ \\
\hline Loggerhead Shrike & $18(6-30)$ & $36(18-54)$ & $34(19-49)$ \\
\hline Warbling Vireo & $40(7-73)$ & $50(16-84)$ & $86(43-130)$ \\
\hline Yellow Warbler & $216(110-322)$ & $193(107-279)$ & $226(114-337)$ \\
\hline Common Yellowthroat & $285(173-398)$ & $196(121-271)$ & $375(235-516)$ \\
\hline Chipping Sparrow & $45(6-83)$ & $105(37-174)$ & $150(44-256)$ \\
\hline Clay-colored Sparrow & $786(523-1,050)$ & $564(334-793)$ & $622(369-875)$ \\
\hline Vesper Sparrow & $414(328-500)$ & $479(374-584)$ & $844(654-1,034)$ \\
\hline Lark Sparrow & $83(16-151)$ & $107(35-178)$ & $89(32-146)$ \\
\hline Lark Bunting & $1,368(974-1,762)$ & $1,541(1,151-1,931)$ & $686(412-960)$ \\
\hline Savannah Sparrow & $1,120(859-1,381)$ & $295(189-400)$ & $596(392-800)$ \\
\hline Baird's Sparrow & $376(208-543)$ & $171(90-251)$ & $279(140-418)$ \\
\hline Grasshopper Sparrow & $661(478-844)$ & $890(648-1,132)$ & $1,001(737-1,265)$ \\
\hline Song Sparrow & $116(64-168)$ & $216(134-298)$ & $274(186-363)$ \\
\hline Chestnut-collared Longspur & $2,544(1,987-3,101)$ & $1,351(913-1,789)$ & $1,707(1,183-2,232)$ \\
\hline Bobolink & $464(313-616)$ & $405(287-523)$ & $371(262-480)$ \\
\hline Red-winged Blackbird & $2,038(1,690-2,386)$ & $1,306(1,021-1,591)$ & $1,536(1,224-1,848)$ \\
\hline Western Meadowlark & $2,034(1,802-2,267)$ & $1,080(911-1,248)$ & $1,441(1,231-1,651)$ \\
\hline Yellow-headed Blackbird & $193(37-350)$ & $340(114-566)$ & $373(183-563)$ \\
\hline Brewer's Blackbird & $43(12-73)$ & $102(22-182)$ & $187(89-285)$ \\
\hline Common Grackle & $302(193-412)$ & $658(400-916)$ & $651(346-957)$ \\
\hline Brown-headed Cowbird & $1,005(794-1,217)$ & $1,425(1,080-1,771)$ & $1,335(1,116-1,554)$ \\
\hline Orchard Oriole & $39(15-64)$ & $73(40-105)$ & $87(51-122)$ \\
\hline Baltimore Oriole & $44(18-71)$ & $105(63-146)$ & $86(45-128)$ \\
\hline American Goldfinch & $227(156-297)$ & $316(193-438)$ & $282(203-361)$ \\
\hline House Sparrow & $214(88-339)$ & $397(208-586)$ & $380(180-580)$ \\
\hline
\end{tabular}

